\title{
VLBI for Gravity Probe B. III. A Limit on the Proper Motion of the "Core" of the Quasar 3C 454.3
}

\section{Citation}

Bartel, N., M. F. Bietenholz, D. E. Lebach, J. I. Lederman, L. Petrov, R. R. Ransom, M. I. Ratner, and I. I. Shapiro. 2012. "VLBI for Gravity PRobe B. III. A Limit on the Proper Motion of the "Core" of the Quasar 3C 454.3." The Astrophysical Journal Supplement Series 201, no. 1:1-20.

\section{Published Version}

doi:10.1088/0067-0049/201/1/3

\section{Permanent link}

http://nrs.harvard.edu/urn-3:HUL.InstRepos:11858832

\section{Terms of Use}

This article was downloaded from Harvard University's DASH repository, and is made available under the terms and conditions applicable to Other Posted Material, as set forth at http:// nrs.harvard.edu/urn-3:HUL.InstRepos:dash.current.terms-of-use\#LAA

\section{Share Your Story}

The Harvard community has made this article openly available.

Please share how this access benefits you. Submit a story.

\section{Accessibility}




\title{
VLBI FOR GRAVITY PROBE B. III. A LIMIT ON THE PROPER MOTION OF THE “CORE” OF THE QUASAR 3C 454.3
}

\author{
N. BARTEL ${ }^{1}$, M. F. Bietenholz ${ }^{1,5}$, D. E. LeBACH ${ }^{2}$, J. I. LeDERMAN ${ }^{3}$, \\ L. Petrov ${ }^{4}$, R. R. RANSOM ${ }^{1,6}$, M. I. RATNeR ${ }^{2}$, AND I. I. ShaPIRO ${ }^{2}$ \\ ${ }^{1}$ Department of Physics and Astronomy, York University, 4700 Keele Street, Toronto, ON M3J 1P3, Canada \\ ${ }^{2}$ Harvard-Smithsonian Center for Astrophysics, 60 Garden Street, Cambridge, MA 02138, USA \\ ${ }^{3}$ Centre for Research in Earth and Space Sciences, York University, 4700 Keele Street, Toronto, ON M3J 1P3, Canada \\ ${ }^{4}$ Astrogeo Center, 7312 Sportsman Drive, Falls Church, VA 22043, USA \\ Received 2011 May 31; accepted 2012 April 5; published 2012 June 7
}

\begin{abstract}
We made very long baseline interferometry observations at $8.4 \mathrm{GHz}$ between 1997 and 2005 to estimate the

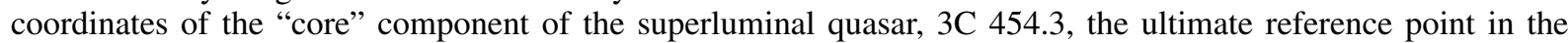
distant universe for the NASA/Stanford Gyroscope Relativity Mission, Gravity Probe B $(G P-B)$. These coordinates are determined relative to those of the brightness peaks of two other compact extragalactic sources, B2250+194 and B2252+172, nearby on the sky, and within a celestial reference frame (CRF), defined by a large suite of compact extragalactic radio sources, and nearly identical to the International Celestial Reference Frame 2 (ICRF2). We find that B2250+194 and B2252+172 are stationary relative to each other, and also in the CRF, to within $1 \sigma$ upper limits of 15 and $30 \mu \mathrm{as} \mathrm{yr}^{-1}$ in $\alpha$ and $\delta$, respectively. The core of 3C 454.3 appears to jitter in its position along the jet direction over $\sim 0.2$ mas, likely due to activity close to the putative supermassive black hole nearby, but on average

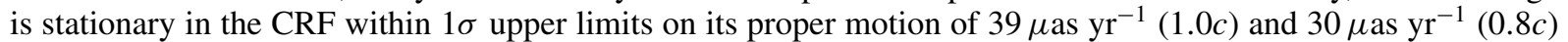
in $\alpha$ and $\delta$, respectively, for the period 2002-2005. Our corresponding limit over the longer interval, 1998-2005, of more importance to $G P-B$, is 46 and $56 \mu \mathrm{as} \mathrm{yr}^{-1}$ in $\alpha$ and $\delta$, respectively. Some of 3C 454.3's jet components show significantly superluminal motion with speeds of up to $\sim 200 \mu \mathrm{as} \mathrm{yr}^{-1}$ or $5 c$ in the CRF. The core of 3C 454.3 thus provides for $G P-B$ a sufficiently stable reference in the distant universe.
\end{abstract}

Key words: binaries: close - radio continuum: stars - stars: activity - stars: imaging - stars: individual (IM Pegasi) - techniques: interferometric

\section{INTRODUCTION}

Gravity Probe $B(G P-B)$ is the space-borne relativity experiment developed by NASA and Stanford University to test two predictions of general relativity. The experiment used four superconducting gyroscopes, contained in a low-altitude, polar orbiting spacecraft, to measure the geodetic effect and the much smaller frame-dragging effect. According to general relativity, each of these effects induces precessions of the gyroscopes in planes perpendicular to each other. For the geodetic effect, which depends directly on Earth's mass, the predicted precession is $6.6 \operatorname{arcsec} \mathrm{yr}^{-1}$ and for the frame-dragging effect, which depends directly on the angular momentum of the Earth, it is

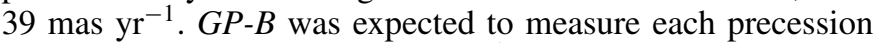

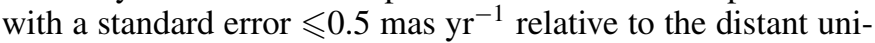
verse. Because of technical limitations, the spacecraft could not measure the precessions directly relative to the distant universe but only to an optically bright star, the guide star, chosen to be IM Pegasi (HR 8703). We must therefore determine IM Peg's proper motion relative to the distant universe, which is, for our purposes, best represented by extragalactic radio sources.

For our part of the $G P-B$ project, we determined the coordinates and the proper motion of the guide star in the radio relative to the "core" of the quasar 3C 454.3 (B2251+158). This core was tied to two other radio sources, which are compact,

\footnotetext{
5 Now also at Hartebeesthoek Radio Astronomy Observatory, PO Box 443, Krugersdorp 1740, South Africa.

6 Now at Okanagan College, 583 Duncan Avenue West, Penticton, BC V2A 2K8, Canada, and also at the National Research Council of Canada, Herzberg Institute of Astrophysics, Dominion Radio Astrophysical Observatory, PO Box 248, Penticton, BC V2A 6K3, Canada.
}

extragalactic, nearby to it on the sky, and also tied to a celestial reference frame (CRF) defined by a large suite of extragalactic sources. These ties are the main subjects of this paper. Most important for $G P-B$, of course, is the bound that we place on the proper motion of the core, which serves as the principal reference for determining the proper motion of IM Peg. The $G P-B$ project needs the proper motion of the optical source in IM Peg. The radio source in IM Peg, however, moves erratically with respect to the optical source. In order to be able to average as well as feasible over the erratic motion, we place our very long baseline interferometry (VLBI) limit on the motion of the core of 3C 454.3 over as long a period as feasible. By contrast, for astrophysical purposes, we place a more stringent bound on the core's motion, but only for a substantially shorter period of time.

Apart from its relevance for $G P-B$, our observations and astrometric analysis are also of astrophysical interest. The quasar 3C 454.3 is a highly active superluminal radio source (see, e.g., Pauliny-Toth et al. 1987). It consists of a relatively compact region from which a bent jet emanates (e.g., PaulinyToth 1998). Superluminal motion refers to those apparent transverse velocities of the components within the source that are measured to be greater than $c$, the speed of light. On the basis of synchrotron radiation theory, the core is generally identified as that component which is compact and has a flat or inverted radio spectrum. Observations at 43 and $86 \mathrm{GHz}$ show that 3C 454.3 has such a component located in the eastern part of the brightness distribution (Pagels et al. 2004). Its characteristics are consistent with those expected for the environment of a supermassive black hole or the base of an associated jet, but not conclusively diagnostic of either one. An additionally powerful probe for the location of a possible supermassive black hole 
in an extragalactic source is to identify the component in the radio structure of the source that shows the smallest motion of all components or is stationary on the sky. Such a component would be a strong candidate for being closely related to the purported supermassive black hole which is likely to be both close to the center of mass of the source and virtually stationary on the sky.

Placement of stringent limits on the proper motions of quasars and other compact extragalactic radio sources distributed across the sky are being made by others on a routine basis through astrometric/geodetic VLBI observations. ${ }^{7}$ Random errors of frequently observed sources may be as low as $6 \mu$ as, although systematic errors mainly due to unaccounted propagation effects and source structure are believed to be in the range of 50-1000 $\mu$ as.

All geodetic VLBI measurements are based on interferometric group delays. More accurate measurements can be made, for example, with targeted differential VLBI observations of two or more sources located close to each other on the sky (Shapiro et al. 1979). In such cases, interferometric phases or phase delays can be used, yielding relative positions and proper motions of properly selected celestial sources with uncertainties as low as $\sim 10 \mu$ as and $\sim 10 \mu$ as $_{\mathrm{yr}^{-1}}$, respectively (e.g., Marcaide \& Shapiro 1983; Bartel et al. 1986; Rioja \& Porcas 2000; Bietenholz et al. 2001; Fomalont \& Kopeikin 2003; Brunthaler et al. 2005).

This paper is the third in a series of seven papers reporting on the astrometric support for $G P-B$ for the purpose of defining the cosmological reference frame for the gyroscope precession measurements. In the first paper of this series we gave an overview of the astronomical support for $G P-B$ (Shapiro et al. 2012, Paper I). In the second paper we focused on the characteristics of quasar 3C 454.3 and the other two extragalactic reference sources, B2250+194 and B2252+172, and reported on their structure and structure changes with time and frequency (Ransom et al. 2012a, Paper II). In this paper (Paper III), we report on the degree of stationarity of the core of the quasar 3C 454.3, which is the reference source for the guide star IM Peg and therefore pivotal for $G P-B$. In the fourth paper we present our VLBI astrometry analysis technique and compare it with other such techniques (Lebach et al. 2012, Paper IV). In the fifth paper we present our results for the proper motion and parallax of IM Peg relative to the core of 3C 454.3 (Ratner et al. 2012, Paper V). In the sixth paper we report on the orbital motion of IM Peg and interpret the radio structure of the star (Ransom et al. 2012b, Paper VI). Finally, in the seventh paper, we focus on the individual epochs of observation of IM Peg and include a movie of the radio images of this star (Bietenholz et al. 2012, Paper VII).

Here we first briefly describe our observations in Section 2. We give characteristics and show representative images of 3C 454.3, B2250+194, and B2252+172 in Section 3. We describe our astrometry program in Section 4 . We present astrometric results in Sections 5-7, discuss these results in Section 8, and give our conclusions in Section 9.

\section{OBSERVATIONS}

As one of the strongest-emitting quasars at radio frequencies, 3C 454.3 has been observed in geodetic group-delay VLBI sessions since 1979 . For our $G P-B$ VLBI program we made use of observations from the total of 1119 such sessions between

\footnotetext{
7 Apart from source positions, these observations yield antenna coordinates and velocities, and a series of Earth orientation parameters.
}

Table 1

Characteristics of the Sources

\begin{tabular}{lcrrcccc}
\hline \hline Source & Type & \multicolumn{2}{c}{ Separation } & Flux Density & Redshift & $\begin{array}{c}\text { Distance } \\
\text { b }\end{array}$ \\
\cline { 3 - 5 } & & $\Delta \alpha\left(^{\circ}\right)$ & $\Delta \delta\left(^{\circ}\right)$ & $(\mathrm{Jy})$ & & $(\mathrm{Mpc})$ \\
\hline 3C 454.3 & Quasar & $\ldots$ & $\ldots$ & $7-10$ & 0.859 & 1610 \\
B2250+194 & Galaxy & -0.2 & 3.6 & $0.35-0.45$ & 0.28 & 880 \\
B2252+172 & Unidentified & 0.4 & 1.4 & 0.017 & $\ldots$ & $\ldots$ \\
IM Peg & RS CVn & -0.1 & 0.7 & $0.005-0.05$ & 0.0 & 0.0 \\
\hline
\end{tabular}

Notes.

a The range gives the lowest and highest flux density we measured at $8.4 \mathrm{GHz}$ with the VLA during the course of our observations, 1997 January to 2005 July. b The angular diameter distance for a flat universe with Hubble constant, $H_{0}=$ $70 \mathrm{~km} \mathrm{~s}^{-1} \mathrm{Mpc}^{-1}$, and normalized density parameters, $\Omega_{M}=0.27$ and $\Omega_{\lambda}=$ 0.73 (Kantowski et al. 2000).

1980 and 2008. In addition we used geodetic observations of B2250+194 from a total of 38 sessions between 1996 and 2008 that were made in support of $G P-B$ VLBI.

The bulk of our $G P-B$ VLBI efforts were devoted to phasedelay VLBI observations of IM Peg and our three reference sources, 3C 454.3, B2250+194, and B2252+172. A detailed description of these latter observations was given in Paper II; however, for the convenience of the reader, we give a summary here.

We obtained 35 sets of $8.4 \mathrm{GHz}$ VLBI observations in support of $G P-B$ between 1997 January 16 and 2005 July 16 . We used a global array of 12-16 radio telescopes, which most often included MPIfR's 100 m telescope at Effelsberg, Germany; NASA/Caltech/JPL's 70 m DSN telescopes at Robledo, Spain, Goldstone, CA, and Tidbinbilla, Australia; NRAO's ten $25 \mathrm{~m}$ telescopes of the Very Long Baseline Array (VLBA), across the US; NRAO's phased Very Large Array (VLA), equivalent to a $130 \mathrm{~m}$ telescope, near Socorro, NM; and, at early times, NRCan's 46 m Algonquin Radio Telescope near Pembroke, ON, Canada, and, at later times, NRAO's 110 m Green Bank Telescope in WV. In each session we made interleaved observations of 3C 454.3, IM Peg, and B2250+194 by using a sequence of typically 3C 454.3 (80 s)-IM Peg (170 s)-2250 (80 s). For the last 12 sessions, starting on 2002 November, we also observed B2252+172, but only after every second sequence to allow greater concentration on the three main sources. The new observing sequence was 3C 454.3 (80 s)-IM Peg (125 s)-2250 (80 s)-3C 454.3 (80 s)-IM Peg (125s)-2250 (80 s)-2252 (90s). ${ }^{8}$ In three sessions we also observed at 5.0 and $15.0 \mathrm{GHz}$. All observations were recorded in both right and left circular polarizations and processed on the VLBA hardware correlator at Socorro.

\section{THE CELESTIAL REFERENCE SOURCES}

\subsection{Sky Positions and Cosmological Distances}

In Figure 1 we show the positions of 3C 454.3, B2250+194, and $\mathrm{B} 2252+172$ along with that of the $G P-B$ guide star, IM Peg. All four sources are located approximately along a single north-south axis allowing us to make easier use of interpolation to estimate and potentially reduce the contributions of the troposphere and the ionosphere to the total error in determining the sources' relative positions. In Table 1 we give the sky separations of the two extragalactic sources from 3C 454.3,

\footnotetext{
8 Here and hereafter we sometimes use as abbreviations 2250 for B2250+194 and 2252 for B2252+172.
} 


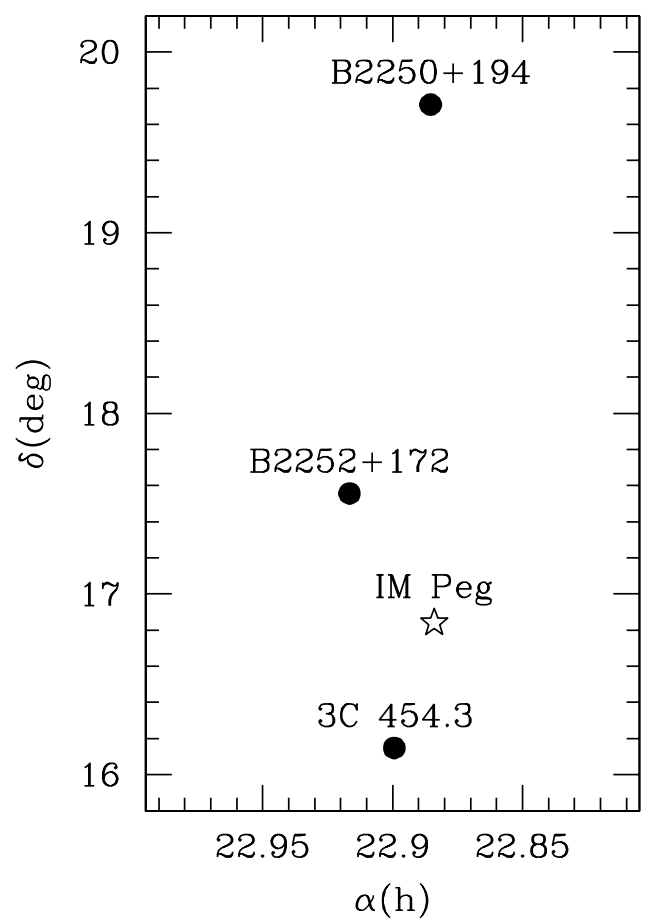

Figure 1. Sky chart with coordinates of the three reference sources and the guide star, IM peg. The linear scale is the same for right ascension $(\alpha)$ and declination $(\delta)$ for the declination of 3 C 454.3 .

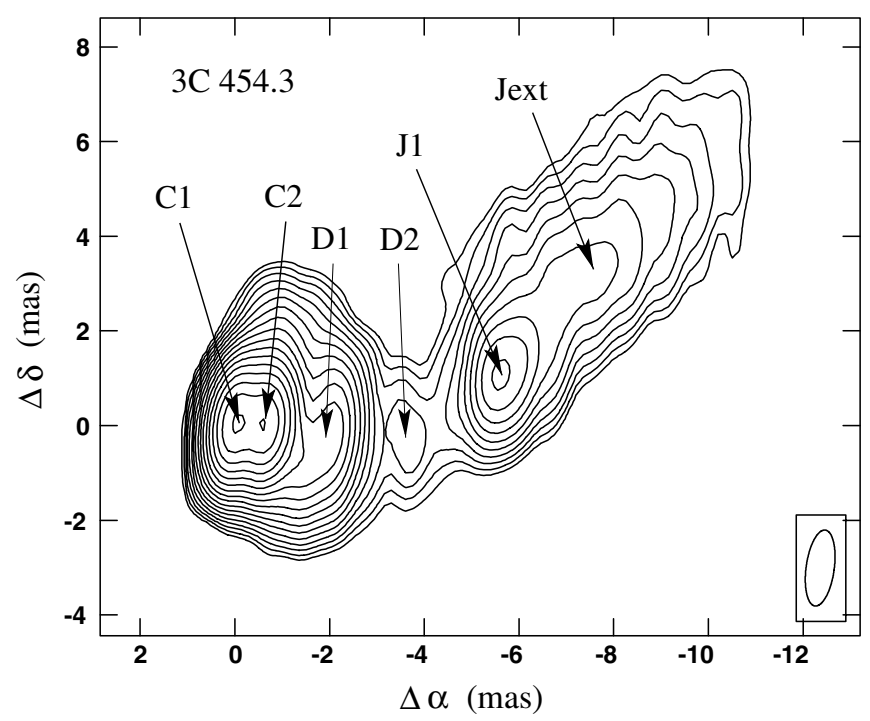

Figure 2. Image of 3C 454.3 from observations on 2005 May 28 with components $\mathrm{C} 1, \mathrm{C} 2, \mathrm{D} 1, \mathrm{D} 2, \mathrm{~J} 1$, and Jext indicated. The contours start at $10 \mathrm{mJy}$ beam ${ }^{-1}$ and increase by factors of $\sqrt{2}$ toward the peak. The peak brightness is $2.68 \mathrm{Jy} \mathrm{beam}^{-1}$. The rms brightness of the background noise is $0.73 \mathrm{mJy}$ beam $^{-1}$. The FWHM contour of the Gaussian convolving beam is given in the lower right. North is up and east is to the left.

the sources' flux densities, redshifts (when known), and angular diameter distances, the latter assuming an inhomogeneous Friedmann-Lemaitre-Robertson-Walker cosmology. For comparison, we also give the characteristics of IM Peg.

\subsection{Representative Images}

In Figures 2-4 we show representative images of 3C 454.3, B2250+194, and B2252+172. The source 3C 454.3 is a superluminal quasar with the highest radio flux density of the three sources. It consists of a core region, which is primarily ex-

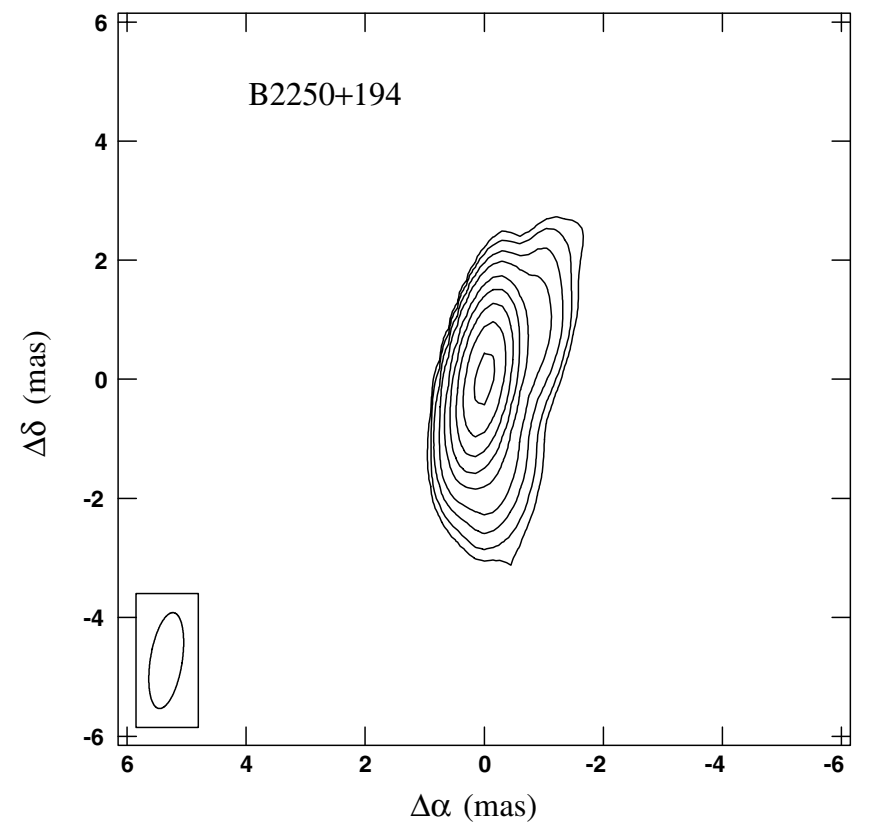

Figure 3. Image of B2250+194 from observations on 2005 January 15. The contours are at $0.3 \%, 0.5 \%, 1 \%, 2 \%, 5 \%, 10 \%, 20 \%, 40 \%$, and $80 \%$ of the peak brightness of $0.43 \mathrm{Jy} \mathrm{beam}^{-1}$. The rms brightness of the background noise is $0.08 \mathrm{mJy}^{\text {beam }}{ }^{-1}$. The FWHM contour of the Gaussian convolving beam is given in the lower left. North is up and east is to the left.

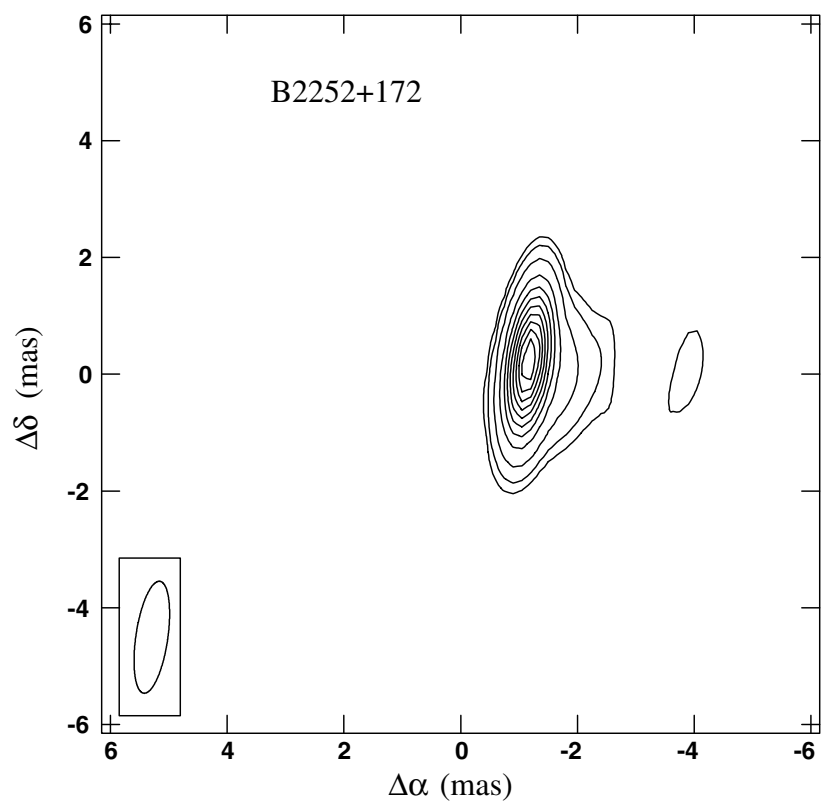

Figure 4. Image of B2252+172 from observations on 2005 January 15 . The contours are at $3 \%, 5 \%, 10 \%, 20 \%, 30 \%, \ldots 90 \%$ of the peak brightness of 0.012 $\mathrm{Jy} \mathrm{beam}^{-1}$. The rms brightness of the background noise is $0.05 \mathrm{mJy}$ beam ${ }^{-1}$. The FWHM contour of the Gaussian convolving beam is given in the lower left. North is up and east is to the left.

tended east-west, and can be well modeled for each of our 35 epochs by two compact components, $\mathrm{C} 1$ and $\mathrm{C} 2$, separated by $\sim 0.6$ mas. When studied at a higher resolution, e.g., at 43 and $86 \mathrm{GHz}$, the same region has a complex structure (Gómez et al. 1999; Jorstad et al. 2001, 2005), with C1 being essentially unresolved and having a size at $86 \mathrm{GHz}$ of $\leqslant 70 \mu$ as (Pagels et al. 2004). C2 is extended. Also, between these two components there are others that appear to move away from $\mathrm{C} 1$ toward $\mathrm{C} 2$ with superluminal speeds (Jorstad et al. 2001, 2005); these other 
components are not individually visible in our lower-resolution $8.4 \mathrm{GHz}$ images.

Further to the west there are components, D1 and D2, that are visible only at later epochs in our observations. We modeled these as compact components. They bridge the core region to the outer part of the jet with a more than 10 mas extent in our images. This outer part of the jet bends toward the northwest and in fact extends as far as a few arcseconds away from the core (see also Cawthorne \& Gabuzda 1996; Pauliny-Toth 1998). The brightness peak of the extended 10 mas jet in our image is clearly visible at each of our 35 epochs, and we modeled it as a compact component which we call J1. The extended jet can be modeled as an elliptical Gaussian, which we call Jext.

The source B2250+194 is 20 times weaker in terms of flux density than $3 \mathrm{C} 454.3$, but much more compact, consisting of a central component with north-south extensions and an apparent length of $\sim 5$ mas. The source B2252+172 is the weakest of the three in terms of flux density but also the most compact, consisting of one dominant component and a barely visible extension to the west. For more detail on the structure of these sources and their evolution or lack thereof, see Paper II.

\subsection{Selection of $3 C 454.3$ as a Reference Source for GP-B}

Because of these differences in characteristics and separations from IM Peg, each of these sources has advantages and disadvantages as a reference source for IM Peg. The quasar 3C 454.3 has the advantage of having the highest flux density and closest proximity to the guide star. The latter point is of chief importance, since the standard errors of astrometric VLBI measurements scale approximately linearly with the separation of the reference source from the target source (Shapiro et al. 1979; Pradel et al. 2006). Another advantage is that 3C 454.3 was used as the astrometric reference source for IM Peg as early as 1991 (Lestrade et al. 1999), thus extending the time baseline of VLBI position determinations and allowing increased accuracy in our proper-motion estimates. The main disadvantage of this reference source is its complex structure.

The advantage of the other two sources is their compact structure. However, B2250+194 is relatively far away on the sky from IM Peg, and B2252+172 has a relatively low flux density. All things considered, we decided to use 3 C 454.3 as the primary reference source for IM Peg. Our task was then to find the component in $3 \mathrm{C} 454.3$ that is most closely associated with the dynamical center of the quasar and to test the stationarity of this component with respect to our two other reference sources and our CRF.

Images of 3C 454.3 at 8.4 and $15 \mathrm{GHz}$ (Paper II) and at 43 and $86 \mathrm{GHz}$ (Pagels et al. 2004) show that the easternmost component, $\mathrm{C} 1$, remains compact at the highest frequencies and angular resolutions yet investigated, and has a flat or inverted spectrum in this frequency region. Other components or condensations show structure at $86 \mathrm{GHz}$, and, in cases where it was determinable, a steep spectral index. These characteristics indicate that for our $8.4 \mathrm{GHz}$ images, $\mathrm{C} 1$ is likely the component most closely related to the putative supermassive black hole and the quasar's center of mass.

\section{THE ASTROMETRY PROGRAM FOR $G P-B$ : GOAL, STRATEGY, AND PROCEDURE}

Goal. Our main goal is to determine C1's position and especially a bound on its proper motion relative to the distant universe, to confirm its suitability as the primary reference for $G P-B$.
Strategy. The distant universe is for our purposes most usefully represented by compact, extragalactic radio sources. We choose a procedure where we first determine the position and proper motion of $\mathrm{C} 1$ relative to our two reference sources, B2250+194 and B2252+172, and second relative to our CRF. For the first step, interferometric phase delays are used exclusively. This has the advantage of simplicity, utilization of the same type of data (phase delays) and analysis technique, and highest precision and possibly highest accuracy of results. For the second, the results from the first are added to position and proper-motion determinations of B2250+194 in the CRF based on interferometric group delays from the geodetic VLBI sessions. This has the advantage of having as a reference not only two very distant and compact sources, but also $\sim 4000$ sources that define our CRF.

Procedure. We determine the position and the limit on proper motion of the following.

1. $\mathrm{C} 1$ relative to $\mathrm{B} 2250+194$ and $\mathrm{B} 2252+172$, and $\mathrm{B} 2252+172$ relative to $\mathrm{B} 2250+194$ in the senses $(\mathrm{C} 1-2250)$, (C1-2252), and (2252-2250) using our interleaved phasedelay VLBI observations. The combined result for $\mathrm{C} 1$ is obtained as a weighted mean of the first two differenced solutions, with the third serving to demonstrate the consistency of our results and zero proper motion within the errors for the two sources relative to each other. Due to the source's compactness, any motions or brightnessdistribution changes of these two reference sources would likely be very small and therefore have only a marginal, if any, effect on our astrometric results for $\mathrm{C} 1$.

2. B2250+194 in the CRF using routine geodetic and astrometric group-delay VLBI observations distributed by the International VLBI Service for Geodesy and Astrometry; ${ }^{9}$

3 . B2252+172 in the CRF by adding the result from (2) to that from (1) in the sense $(2252-2250)+2250$, and confirming the position result and proper-motion limit by using recent single-epoch geodetic group-delay VLBI observations of B2252+172.

4. $\mathrm{C} 1$ in the CRF in two ways: first by adding the results from (1) to those from (2): $\mathrm{C} 1=(\mathrm{C} 1-2250)+2250$, and second by adding the results from (1) to those of (3): $\mathrm{C} 1=$ $(\mathrm{C} 1-2252)+2252$. The combined result is obtained as a weighted mean from these two methods.

\section{ASTROMETRIC RESULTS (1): POSITION DETERMINATIONS FOR EACH OBSERVING SESSION}

\subsection{Analysis of Interleaved Phase-delay Observations of $3 C 454.3, B 2250+194$, and B2252+172}

Our VLBI data for the reference sources, 3C 454.3, B2250+194, B2252+172, and also from IM Peg, were analyzed with an astrometric software package that was developed specifically for the analysis of the $G P-B$ VLBI data. It includes a phase-connection program that automatically resolves $2 \pi$ ambiguities that exist in the set of VLBI phases for each baseline so as to convert them to phase delays. The software also includes in the phase-delay fitting (e.g., Shapiro et al. 1979; Bartel et al. 1986) a Kalman filter (see Paper IV) to model the variations of the troposphere, the ionosphere, and the clock offsets at each VLBI site (Lebach et al. 1999). In addition, we used two different models to initially correct for the effects of the ionosphere, one we call "JPL" which is part of NRAO's imaging package,

\footnotetext{
9 Available at http://ivscc.gsfc.nasa.gov/products-data/index.html.
} 
AIPS, and based on GPS data provided by JPL, and the other, the older parameterized ionospheric model "PIM," developed at USAF Research Labs (described by, e.g., Campbell 1999).

To relate the phase delays to a particular reference point in 3C 454.3, namely the core component $\mathrm{C} 1$, all phase delays from differential astrometry involving 3C 454.3 were corrected for the structure of $3 \mathrm{C} 454.3$, as represented by the CLEAN components produced with AIPS; C1 served as the phase-reference point. The other two extragalactic sources were deemed sufficiently compact for our purposes so that the reference point for each could be assumed to be the brightness peak in its image. We elaborate on this method of astrometric VLBI data analysis and compare it to other methods in Paper IV.

\subsection{Positions of the Components of $3 C 454.3$ Relative to Those of B2250+194 and B2252+172}

To test the positional stability of 3C 454.3's component C1 relative to our two reference sources, we determined, for each of our 35 sessions of $8.4 \mathrm{GHz}$ observations, the coordinates of $\mathrm{C} 1$, and, for comparison, also those of C2, D1, D2, J1, and Jext, all relative to the brightness peak of B2250+194 and, for the last 12 epochs, also to that of B2252+172. We obtained two sets of coordinates by correcting for the effects of the ionosphere in two different ways, one set by using the JPL model and another set by using PIM. Although the JPL model was not available for our first eight epochs but only from 1998 September 17 onward, we nevertheless in this paper use mainly phase-delay data corrected with the JPL model since it proved to be superior in that it resulted in smaller uncertainties of our astrometric estimates despite precluding the use of our earliest phase-delay data. We elaborate on the comparison below. (In Paper V we use PIM instead, because in that paper errors in modeling the ionosphere play a less significant role than they play here, and because PIM has the advantage that it can be used for all of our VLBI data.) We list our coordinate determinations for $\mathrm{C} 1$, $\mathrm{C} 2$, D1, D2, J1, and Jext relative to the brightness peak of B2250+194 in Table 2 and to that of B2252+172 in Table 3, all obtained with the JPL model.

\subsection{The Uncertainties of the Relative Positions of the Components of $3 C 454.3$}

The uncertainties of the coordinates listed in Tables 2 and 3 were determined partly empirically, namely by adding a constant in quadrature to the statistical standard errors so as to obtain a reduced Chi-square of unity $\left(\chi_{v}^{2} / v=1\right.$, where $v$ is the number of degrees of freedom) in our residuals after solving for relative position and proper motion in $\alpha$ and $\delta$ separately. This constant is assumed to approximately reflect nonstatistical errors. Accurate standard errors are difficult to estimate in any other way. They contain contributions from noise and from systematic errors, with the latter due mostly to deconvolution, source structure, and atmospheric and ionospheric variations. We next discuss and approximately quantify each contribution in turn.

\subsubsection{Noise}

Noise in an image has a number of sources. The rms background brightness in the images is dominated by contributions from statistical noise in the radio signals and thermal noise in the receivers. However, for our relatively high dynamic range images (typically over 2000-1; see, e.g., Figures 2-4), the various uncertainties in the bright parts of the image are larger than their corresponding rms background brightnesses and are dominated by contributions which are not strictly random such as residual calibration errors and deconvolution errors. Given the small rms of the background brightness relative to the peaks in the maps, we conclude that this noise causes errors in the estimate of the separation of each of components $\mathrm{C} 1$ and $\mathrm{C} 2$ in $3 \mathrm{C} 454.3$ from B2250+194 by a correspondingly small portion of the half-width at half-maximum (HWHM) of the beam, namely by $<5 \mu$ as, and of each of components D1, D2, J1, and Jext in $3 \mathrm{C} 454.3$ from B2250+194 by $<10 \mu$ as. The corresponding errors in our estimates of the separation of these components from the weaker source, B2252+172, are dominated by the source's lower peak-to-noise ratio but are still $<10 \mu$ as.

\subsubsection{Deconvolution Errors}

Deconvolution errors are caused by the visibility measurements not filling the $u-v$ plane of the VLBI array up to its highest angular resolution and by the resulting generation of side lobes in the image plane, which are not completely eliminated through the deconvolution process. We studied this type of error by using a noise-free model image similar to the image of 3C 454.3 at $8.4 \mathrm{GHz}$, Fourier-transforming the model to the $u-v$ plane, and then using the same $u-v$ plane sampling as in one of our typical observing sessions. The generated $u-v$ model data were then used for imaging and deconvolution. We then determined the difference between the position of each component in the model image and the position of the corresponding component in the deconvolved image. We found that the deconvolution error for each coordinate of each of the six components of $3 \mathrm{C} 454.3$ is typically $30 \mu$ as and never larger than $\sim 40 \mu$ as. The deconvolution errors for sources like B2250+194 and B2252+172 with relatively simple brightness distributions are doubtless smaller given the same $u-v$ coverage as for $3 \mathrm{C} 454.3$. We therefore conclude that the standard error in each coordinate of the separation of any component in 3C 454.3 from either B2250+194 or B2252+172 is typically $30 \mu$ as.

\subsubsection{Structure Errors}

Structure errors are caused by a mismatch between the Gaussian component model and the brightness distribution of the source. If the source were completely unresolved, a fit of a Gaussian to the image with the parameters of the convolving beam would give the position of the source with an essentially zero structure error. Indeed, the sources B2250+194 and $\mathrm{B} 2252+172$ are rather compact, with brightness peaks that can be clearly identified and located, and hence we expect the structure errors to be small in comparison to those for the components in 3C 454.3. As can be seen in Figure 2, components $\mathrm{C} 1$ and $\mathrm{C} 2$ are located close together in comparison to the size of the beam and can barely be distinguished at several epochs (see Paper II). In addition, there is "confusing" emission in the neighborhood of these two components. These two characteristics cause significant structure errors, difficult to estimate quantitatively, but likely as large as a good fraction of the HWHM of the beam. The components D1 and D2 are weaker than $\mathrm{C} 1$ and $\mathrm{C} 2$, and visible only at later epochs, but then clearly distinguishable. Their identification is likely as uncertain as that of $\mathrm{C} 1$ and $\mathrm{C} 2$. Component $\mathrm{J} 1$ is always visible as a single peak; however, it is embedded in the extended brightness distribution of the 10 mas long jet rendering the identification of a component in that jet as uncertain as, for many epochs, the identification of $\mathrm{C} 1$ and $\mathrm{C} 2$. The identification of Jext is more uncertain than that of any of the other components since this component represents 
Table 2

Separation of 3C 454.3 Components from B2250+194

\begin{tabular}{|c|c|c|c|c|c|c|c|c|c|c|c|c|c|}
\hline \multirow[t]{2}{*}{ Epoch } & \multirow{2}{*}{$\begin{array}{l}\text { Julian Date } \\
2450000.0+\end{array}$} & \multicolumn{2}{|c|}{$\mathrm{C}^{\mathrm{a}}$} & \multicolumn{2}{|c|}{$\mathrm{C} 2^{\mathrm{a}}$} & \multicolumn{2}{|c|}{$\mathrm{D} 1^{\mathrm{a}}$} & \multicolumn{2}{|c|}{$\mathrm{D} 2^{\mathrm{a}}$} & \multicolumn{2}{|c|}{$\mathrm{J} 1^{\mathrm{a}}$} & \multicolumn{2}{|c|}{ Jext $^{\mathrm{a}}$} \\
\hline & & $\begin{array}{c}\Delta \alpha \\
\text { (mas) }\end{array}$ & $\begin{array}{c}\Delta \delta \\
(\mathrm{mas})\end{array}$ & $\begin{array}{c}\Delta \alpha \\
(\mathrm{mas})\end{array}$ & $\begin{array}{c}\Delta \delta \\
(\mathrm{mas})\end{array}$ & $\begin{array}{c}\Delta \alpha \\
(\mathrm{mas})\end{array}$ & $\begin{array}{c}\Delta \delta \\
(\mathrm{mas})\end{array}$ & $\begin{array}{c}\Delta \alpha \\
(\mathrm{mas})\end{array}$ & $\begin{array}{c}\Delta \delta \\
\text { (mas) }\end{array}$ & $\begin{array}{c}\Delta \alpha \\
(\mathrm{mas})\end{array}$ & $\begin{array}{c}\Delta \delta \\
\text { (mas) }\end{array}$ & $\begin{array}{c}\Delta \alpha \\
\text { (mas) }\end{array}$ & $\begin{array}{c}\Delta \delta \\
\text { (mas) }\end{array}$ \\
\hline 1998 Sep 17 & 1073.8 & $-0.11 \pm 0.08$ & $0.25 \pm 0.09$ & $-0.58 \pm 0.11$ & $0.22 \pm 0.10$ & & $\ldots$ & $\ldots$ & $\ldots$ & $-5.57 \pm 0.07$ & $0.72 \pm 0.10$ & $-6.34 \pm 0.10$ & $2.09 \pm 0.08$ \\
\hline 1999 Mar 13 & 51.3 & $0.21 \pm 0.08$ & $1 \pm 0.08$ & $0.68 \pm 0.12$ & $.06 \pm 0.09$ & $\ldots$ & $\ldots$ & $\ldots$ & $\ldots$ & $5.63 \pm 0.07$ & $61 \pm 0.09$ & $-6.46 \pm 0.11$ & $.99 \pm 0.08$ \\
\hline 1999 May 15 & 1314.1 & $-0.11 \pm 0.08$ & $14 \pm 0.08$ & $0.52 \pm 0.12$ & $0.07 \pm 0.09$ & $\ldots$ & $\ldots$ & $\ldots$ & $\ldots$ & $-5.48 \pm 0.07$ & $0.64 \pm 0.09$ & $-6.29 \pm 0.11$ & $1.98 \pm 0.08$ \\
\hline 9 Sep 18 & .8 & $0.27 \pm 0.08$ & $3 \pm 0.08$ & $0.68 \pm 0.12$ & $14 \pm 0$ & $\ldots$ & $\ldots$ & $\ldots$ & $\ldots$ & $.66 \pm 0.07$ & $0.74 \pm 0.10$ & $-6.54 \pm 0.11$ & $14 \pm 0.08$ \\
\hline $1999 \operatorname{Dec} 9$ & .5 & $-0.12 \pm 0.08$ & $21 \pm 0.08$ & $0.60 \pm 0.12$ & $16 \pm 0$ & $\ldots$ & $\ldots$ & $\ldots$ & $\ldots$ & $.59 \pm 0.07$ & $0.76 \pm 0.10$ & $-6.52 \pm 0.10$ & $24 \pm 0$ \\
\hline 2000 May 15 & 80.1 & $10 \pm 0$ & $6 \pm 0$ & $0.71 \pm 0.12$ & $7=$ & $\ldots$ & $\ldots$ & $\ldots$ & $\ldots$ & $64 \pm 0.08$ & $87 \pm$ & $-6.50 \pm 0.10$ & $05 \pm$ \\
\hline 2000 Aug 7 & 1 & $.13 \pm 0.09$ & 09 & $0.78 \pm 0.14$ & $0.08 \pm$ & $\ldots$ & $\ldots$ & $\ldots$ & $\ldots$ & $64 \pm 0.09$ & .10 & 0.11 & .09 \\
\hline 2000 Nov 5 & & $.26 \pm 0.08$ & $66 \pm$ & $0.84 \pm 0.13$ & $-0.01 \pm$ & $\ldots$ & $\ldots$ & $\ldots$ & $\ldots$ & $68 \pm 0.08$ & 09 & 0.10 & .08 \\
\hline 2000 Nov 6 & 1 & $-0.44 \pm 0.13$ & $-0.36 \pm 0.18$ & $1.14 \pm 0.16$ & $-0.42 \pm 0.18$ & $\ldots$ & $\ldots$ & $\ldots$ & $\ldots$ & $-5.93 \pm 0.12$ & $0.35 \pm$ & $-7.01 \pm 0.14$ & $2.01 \pm$ \\
\hline 2001 Mar 31 & 00.2 & .09 & 10 & $0.37 \pm 0.12$ & 0. & $\ldots$ & $\ldots$ & $\ldots$ & $\ldots$ & $-5.42 \pm 0.08$ & $0.96=$ & $-6.48 \pm 0.11$ & $2.34 \pm$ \\
\hline 2001 Jun 29 & 90.0 & $0.05 \pm$ & 08 & $0.59 \pm 0.14$ & $09 \pm$ & $\ldots$ & $\ldots$ & $\ldots$ & $\ldots$ & $-5.57 \pm 0.09$ & $0.95 \pm$ & $-6.50 \pm$ & $2.22 \pm$ \\
\hline 2001 Oct 19 & 02.7 & $0.12 \pm 0.11$ & 0.15 & $0.70 \pm 0.14$ & $-0.05 \pm$ & $\ldots$ & $\ldots$ & $\ldots$ & $\ldots$ & $-5.65 \pm 0.11$ & $0.88 \pm$ & $-6.68 \pm 0.13$ & $2.29 \pm$ \\
\hline 2001 Dec 21 & 65.5 & $0.21 \pm 0.08$ & $-0.06 \pm 0.08$ & $0.89 \pm 0.14$ & $-0.13 \pm 0.09$ & $\ldots$ & $\ldots$ & $\ldots$ & $\ldots$ & $-5.75 \pm 0.08$ & $0.79 \pm 0.09$ & $-6.81 \pm 0.11$ & $2.28 \pm$ \\
\hline 2002 Apr 14 & 79.2 & $0.09 \pm 0.09$ & $0.12 \pm 0.11$ & $-0.64 \pm 0.12$ & $0.01 \pm 0.12$ & 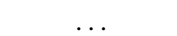 & $\ldots$ & $\ldots$ & $\ldots$ & $-5.58 \pm 0.09$ & $1.06 \pm 0.13$ & $-6.65 \pm 0.11$ & $2.41 \pm 0.11$ \\
\hline 2002 Jul 14 & 69.9 & $0.09 \pm 0.08$ & $10 \pm 0.09$ & $-0.60 \pm 0.14$ & $00 \pm 0.10$ & $\ldots$ & $\ldots$ & $\ldots$ & $\ldots$ & $-5.59 \pm 0.08$ & $0.98 \pm 0.09$ & $-6.65 \pm 0.11$ & $2.34 \pm$ \\
\hline 2002 Nov 20 & 99.6 & $0.12 \pm 0.09$ & $-0.16 \pm 0.10$ & $-0.69 \pm 0.13$ & $-0.25 \pm 0.11$ & $\ldots$ & $\ldots$ & $\ldots$ & $\ldots$ & $-5.71 \pm 0.09$ & $0.77 \pm 0.11$ & $-6.79 \pm 0.11$ & $2.22 \pm$ \\
\hline 2003 Jan 26 & 66.4 & $-0.08 \pm 0.09$ & $0.06 \pm 0.10$ & $-0.67 \pm 0.13$ & $-0.06 \pm 0.11$ & $-1.56 \pm 0.07$ & $-0.20 \pm 0.07$ & $\ldots$ & $\ldots$ & $-5.65 \pm 0.09$ & $0.95 \pm 0.11$ & $-6.67 \pm 0.11$ & $2.28 \pm$ \\
\hline 03 May 18 & 78. & 0.08 & .08 & $0.64 \pm 0.12$ & $-0.01 \pm 0.09$ & $.51 \pm 0.08$ & $24 \pm$ & $\ldots$ & $\ldots$ & $-5.64 \pm 0.08$ & $1.03 \pm$ & $-6.59 \pm 0.10$ & $2.30=$ \\
\hline 2003 Sep 8 & & $2 \pm 0.08$ & $2 \pm 0.09$ & $-0.69 \pm 0.12$ & $-0.06 \pm 0.10$ & $-1.62 \pm 0.08$ & $-0.30 \pm$ & $\ldots$ & $\ldots$ & $-5.60 \pm 0.11$ & $1.00 \pm 0.11$ & $-6.61 \pm 0.11$ & $2.28 \pm 0.09$ \\
\hline $2003 \operatorname{Dec} 5$ & 79.5 & $-0.01 \pm 0.08$ & $11 \pm 0.09$ & $-0.73 \pm 0.11$ & $0.01 \pm 0.10$ & $-1.70 \pm 0.09$ & $-0.27 \pm 0.06$ & $\ldots$ & $\ldots$ & $-5.59 \pm 0.08$ & $1.03 \pm 0.10$ & $-6.63 \pm 0.10$ & $2.37 \pm 0.08$ \\
\hline 2004 Mar 6 & 071.3 & $0.02 \pm 0.08$ & $.00 \pm 0.09$ & $-0.68 \pm 0.12$ & $-0.10 \pm 0.10$ & $-1.66 \pm 0.09$ & $-0.37 \pm 0.05$ & $-3.53 \pm 0.05$ & $-0.50 \pm 0.13$ & $-5.56 \pm 0.08$ & $0.96 \pm 0.10$ & $-6.62 \pm 0.10$ & $2.38 \pm 0.08$ \\
\hline 2004 May 18 & 3144.1 & $-0.03 \pm 0.09$ & $0.09 \pm 0.09$ & $-0.71 \pm 0.12$ & $-0.06 \pm 0.10$ & $-1.69 \pm 0.07$ & $-0.29 \pm 0.07$ & $-3.59 \pm 0.05$ & $-0.41 \pm 0.12$ & $-5.57 \pm 0.10$ & $1.05 \pm 0.12$ & $-6.72 \pm 0.11$ & $2.53 \pm 0.09$ \\
\hline 2004 Jun 26 & 183.0 & $-0.13 \pm 0.08$ & $-0.04 \pm 0.09$ & $-0.83 \pm 0.16$ & $-0.18 \pm 0.10$ & $-1.86 \pm 0.07$ & $-0.37 \pm 0.06$ & $-3.69 \pm 0.05$ & $-0.53 \pm 0.10$ & $-5.61 \pm 0.10$ & $0.91 \pm 0.10$ & $-6.73 \pm 0.11$ & $2.31 \pm 0.08$ \\
\hline 2004 Dec 11 & 351.5 & $0.01 \pm 0.08$ & $0.01 \pm 0.08$ & $-0.67 \pm 0.12$ & $-0.06 \pm 0.09$ & $-1.66 \pm 0.10$ & $-0.44 \pm 0.05$ & $-3.70 \pm 0.04$ & $-0.39 \pm 0.10$ & $-5.62 \pm 0.09$ & $0.94 \pm 0.09$ & $-6.77 \pm 0.10$ & $2.45 \pm 0.07$ \\
\hline 2005 Jan 15 & 3386.4 & $.06 \pm 0.08$ & $-0.05 \pm 0.08$ & $-0.62 \pm 0.12$ & $-0.10 \pm 0.09$ & $-1.66 \pm 0.10$ & $-0.48 \pm 0.05$ & $-3.64 \pm 0.05$ & $-0.55 \pm 0.10$ & $-5.57 \pm 0.08$ & $0.88 \pm 0.09$ & $-6.75 \pm 0.10$ & $2.42 \pm 0.08$ \\
\hline 2005 May 28 & 3519.1 & $0.00 \pm 0.08$ & $0.04 \pm 0.08$ & $-0.69 \pm 0.12$ & $0.00 \pm 0.09$ & $-1.82 \pm 0.09$ & $-0.40 \pm 0.05$ & $-3.71 \pm 0.05$ & $-0.23 \pm 0.10$ & $-5.63 \pm 0.08$ & $1.02 \pm 0.10$ & $-6.75 \pm 0.10$ & $2.52 \pm 0.08$ \\
\hline $2005 \mathrm{Jul} 16$ & 3567.9 & $-0.04 \pm 0.08$ & $0.09 \pm 0.09$ & $-0.76 \pm 0.12$ & $0.06 \pm 0.10$ & $-1.92 \pm 0.09$ & $-0.34 \pm 0.06$ & $-3.74 \pm 0.05$ & $-0.23 \pm 0.11$ & $-5.69 \pm 0.08$ & $1.05 \pm 0.11$ & $-6.86 \pm 0.10$ & $2.60 \pm 0.08$ \\
\hline
\end{tabular}

Notes. ${ }^{\text {a }}$ The coordinate differences of the components of 3C 454.3 from those of B2250+194 (3C 454.3-2250) for each epoch for which the JPL model for the correction of the ionospheric effects could be used: $00^{\mathrm{h}} 00^{\mathrm{m}} 50^{\mathrm{s}} 3787837+\Delta \alpha$ and $-3^{\circ} 33^{\prime} 41^{\prime \prime} 067505+\Delta \delta$. The coordinate differences are based on our differential measurements of C1 relative to B2250+194 and on the determinations of C2, D1, D2, J1, and Jext relative to C1 (Paper II). For B2250+194 the CRF coordinates $22^{\mathrm{h}} 53^{\mathrm{m}} 7^{\mathrm{s}} .3691736$ and $19^{\circ} 42^{\prime} 34^{\prime \prime} .628786$ (solution no. 3, Table 5) were used. The standard errors are the statistical standard errors with a constant added in quadrature so that $\chi_{v}^{2} / v=1$. 


\begin{tabular}{|c|c|c|c|c|c|c|c|c|c|c|c|c|c|}
\hline \multirow[t]{2}{*}{ Epoch } & \multirow{2}{*}{$\begin{array}{c}\text { Julian } \\
\text { Date } \\
2450000+\end{array}$} & \multicolumn{2}{|c|}{$\mathrm{C}^{\mathrm{a}}$} & \multicolumn{2}{|c|}{$\mathrm{C} 2^{\mathrm{a}}$} & \multicolumn{2}{|c|}{$\mathrm{D} 1^{\mathrm{a}}$} & \multicolumn{2}{|c|}{$\mathrm{D} 2^{\mathrm{a}}$} & \multicolumn{2}{|c|}{$\mathrm{J} 1^{\mathrm{a}}$} & \multicolumn{2}{|c|}{ Jext $^{\mathrm{a}}$} \\
\hline & & $\begin{array}{c}\Delta \alpha \\
(\mathrm{mas})\end{array}$ & $\begin{array}{c}\Delta \delta \\
(\mathrm{mas})\end{array}$ & $\begin{array}{c}\Delta \alpha \\
(\mathrm{mas})\end{array}$ & $\begin{array}{c}\Delta \delta \\
(\mathrm{mas})\end{array}$ & $\begin{array}{c}\Delta \alpha \\
(\mathrm{mas})\end{array}$ & $\begin{array}{c}\Delta \delta \\
(\mathrm{mas})\end{array}$ & $\begin{array}{c}\Delta \alpha \\
(\mathrm{mas})\end{array}$ & $\begin{array}{c}\Delta \delta \\
(\mathrm{mas})\end{array}$ & $\begin{array}{c}\Delta \alpha \\
(\mathrm{mas})\end{array}$ & $\begin{array}{c}\Delta \delta \\
(\mathrm{mas})\end{array}$ & & \\
\hline 2002 Nov 20 & 2599.6 & $-0.11 \pm 0.05$ & $-0.06 \pm 0.04$ & $-0.68 \pm 0.06$ & $-0.15 \pm 0.05$ & & & & & $-5.70 \pm 0.05$ & $0.87 \pm 0.04$ & $-6.78 \pm 0.07$ & $2.32 \pm 0.07$ \\
\hline 2003 Jan 26 & 2666.4 & $-0.05 \pm 0.05$ & $0.07 \pm 0.04$ & $-0.64 \pm 0.07$ & $-0.06 \pm 0.05$ & $-1.53 \pm 0.05$ & $-0.20 \pm 0.06$ & & & $-5.62 \pm 0.06$ & $0.95 \pm 0.05$ & $-6.64 \pm 0.07$ & $2.28 \pm 0.07$ \\
\hline 2003 May 18 & 2778.1 & $0.04 \pm 0.05$ & $-0.02 \pm 0.04$ & $-0.65 \pm 0.03$ & $-0.09 \pm 0.04$ & $-1.53 \pm 0.07$ & $-0.32 \pm 0.05$ & & & $-5.65 \pm 0.01$ & $0.96 \pm 0.05$ & $-6.61 \pm 0.06$ & $2.23 \pm 0.06$ \\
\hline 2003 Sep 8 & 2891.7 & $0.02 \pm 0.05$ & $0.01 \pm 0.04$ & $-0.69 \pm 0.04$ & $-0.08 \pm 0.04$ & $-1.62 \pm 0.06$ & $-0.32 \pm 0.05$ & & & $-5.61 \pm 0.08$ & $0.99 \pm 0.05$ & $-6.61 \pm 0.06$ & $2.27 \pm 0.07$ \\
\hline 2003 Dec 5 & 2979.5 & $-0.02 \pm 0.05$ & $0.00 \pm 0.04$ & $-0.74 \pm 0.03$ & $-0.10 \pm 0.05$ & $-1.71 \pm 0.07$ & $-0.37 \pm 0.05$ & $\ldots$ & $\ldots$ & $-5.60 \pm 0.04$ & $0.93 \pm 0.05$ & $-6.64 \pm 0.06$ & $2.27 \pm 0.07$ \\
\hline 2004 Mar 6 & 3071.3 & $-0.01 \pm 0.05$ & $-0.02 \pm 0.04$ & $-0.70 \pm 0.03$ & $-0.12 \pm 0.05$ & $-1.69 \pm 0.07$ & $-0.39 \pm 0.05$ & $-3.56 \pm 0.03$ & $-0.52 \pm 0.11$ & $-5.59 \pm 0.03$ & $0.93 \pm 0.05$ & $-6.64 \pm 0.06$ & $2.36 \pm 0.07$ \\
\hline 2004 May 18 & 3144.1 & $-0.06 \pm 0.05$ & $0.06 \pm 0.05$ & $-0.74 \pm 0.05$ & $-0.08 \pm 0.05$ & $-1.73 \pm 0.06$ & $-0.32 \pm 0.05$ & $-3.62 \pm 0.03$ & $-0.43 \pm 0.10$ & $-5.61 \pm 0.07$ & $1.03 \pm 0.07$ & $-6.76 \pm 0.06$ & $2.50 \pm 0.07$ \\
\hline 2004 Jun 26 & 3183.0 & $-0.09 \pm 0.05$ & $-0.04 \pm 0.04$ & $-0.79 \pm 0.11$ & $-0.18 \pm 0.05$ & $-1.82 \pm 0.05$ & $-0.38 \pm 0.05$ & $-3.65 \pm 0.02$ & $-0.53 \pm 0.08$ & $-5.57 \pm 0.06$ & $0.90 \pm 0.04$ & $-6.69 \pm 0.06$ & $2.30 \pm 0.06$ \\
\hline 2004 Dec 11 & 3351.5 & $0.02 \pm 0.05$ & $-0.04 \pm 0.04$ & $-0.66 \pm 0.04$ & $-0.11 \pm 0.04$ & $-1.65 \pm 0.08$ & $-0.49 \pm 0.05$ & $-3.69 \pm 0.02$ & $-0.45 \pm 0.08$ & $-5.61 \pm 0.05$ & $0.89 \pm 0.05$ & $-6.76 \pm 0.06$ & $2.40 \pm 0.06$ \\
\hline 2005 Jan 15 & 3386.4 & $0.04 \pm 0.05$ & $-0.02 \pm 0.04$ & $-0.64 \pm 0.04$ & $-0.08 \pm 0.04$ & $-1.69 \pm 0.09$ & $-0.46 \pm 0.05$ & $-3.66 \pm 0.03$ & $-0.52 \pm 0.08$ & $-5.59 \pm 0.04$ & $0.91 \pm 0.04$ & $-6.77 \pm 0.06$ & $2.45 \pm 0.06$ \\
\hline 2005 May 28 & 3519.1 & $0.00 \pm 0.05$ & $0.03 \pm 0.04$ & $-0.70 \pm 0.03$ & $-0.01 \pm 0.04$ & $-1.83 \pm 0.08$ & $-0.41 \pm 0.05$ & $-3.72 \pm 0.03$ & $-0.24 \pm 0.08$ & $-5.64 \pm 0.03$ & $1.01 \pm 0.04$ & $-6.76 \pm 0.06$ & $2.51 \pm 0.06$ \\
\hline 2005 Jul 16 & 3567.9 & $0.00 \pm 0.05$ & $0.02 \pm 0.04$ & $-0.73 \pm 0.03$ & $-0.01 \pm 0.04$ & $-1.89 \pm 0.07$ & $-0.41 \pm 0.05$ & $-3.71 \pm 0.02$ & $-0.30 \pm 0.09$ & $-5.66 \pm 0.03$ & $0.97 \pm 0.06$ & $-6.82 \pm 0.06$ & $2.52 \pm 0.06$ \\
\hline
\end{tabular}

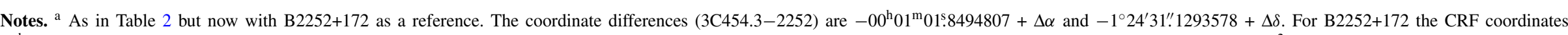
$22^{\mathrm{h}} 54^{\mathrm{m}} 59^{\mathrm{s}} .5974430$ and $17^{\circ} 33^{\prime} 24^{\prime \prime} .690713$ (solution no. 6, Table 5) were used. The standard errors are the statistical standard errors with a constant added in quadrature so that $\chi_{\nu}^{2} / \nu=1$. 
the extended brightness distribution that $\mathrm{J} 1$ is embedded in. The extension is largely toward the northwest, thus affecting both position coordinates of Jext. A detailed analysis of these uncertainties is difficult to carry out quantitatively, in view of the unknown characteristics of the relevant structures. Based on our experience, however, we expect structure errors in each of the position coordinates to be $\sim 30 \mu$ as for components $\mathrm{C} 1$, $\mathrm{C} 2$, D1, D2, and $\mathrm{J} 1$ and $\sim 50 \mu$ as for the component Jext. In comparison, the structure errors for B2250+194 and B2252+172 are negligible.

\subsubsection{Residual Propagation-medium Errors}

Using our Kalman filter (Lebach et al. 2012, Paper IV) removes a large portion of the propagation-medium errors from the estimates of separation between two sources. Nevertheless, errors in modeling the troposphere and the ionosphere at each site likely still represent the largest sources of error in our estimates of relative positions. These errors scale approximately with the separation between the sources (Shapiro et al. 1979; Pradel et al. 2006). These errors also depend on the model used for the ionosphere (JPL or PIM).

In our case the sources are oriented approximately north-south with the separations in $\alpha$ of B2250+194 and $\mathrm{B} 2252+172$ from $\mathrm{C} 1$ being about equal, but with the separation in $\delta$ of $\mathrm{B} 2252+172$ from $\mathrm{C} 1$ being only about $40 \%$ of that for $\mathrm{B} 2250+194$ from $\mathrm{C} 1$. Also, the position determinations are more affected in $\delta$ due to the propagation medium's distorting effects depending strongly on the elevation angle of the source. Therefore, any residual uncorrected ionospheric or tropospheric effects should be most visible in comparisons of the estimated declinations of $\mathrm{C} 1$ relative to $\mathrm{B} 2250+194$ with those relative to $\mathrm{B} 2252+172$, since the angular separation of B2250+194 and B2252+172 is predominantly north-south. To evaluate the adequacy of the models, we plot the relative coordinates, $\mathrm{C} 1-\mathrm{B} 2250+194$, as a function of the relative coordinates, $\mathrm{C} 1-\mathrm{B} 2252+172$, separately in $\alpha$ and $\delta$. For instance, a straight-line slope significantly larger than unity would indicate uncorrected ionospheric or tropospheric effects. We plot the relative coordinates for the JPL model and for PIM in Figure 5. The data corrected with the JPL model were taken from Tables 2 and 3, whereas the data corrected with PIM are not listed.

Least-squares fits ${ }^{10}$ to the data give slopes that are all larger than unity, reflecting the larger separation of B2250+194 from $\mathrm{C} 1$ than of $\mathrm{B} 2252+172$ from $\mathrm{C} 1$. However, the slopes are within $1.3 \sigma$ of unity, except for the data in $\delta$ for the PIM model. Here the slope is $1.78 \pm 0.20$. It is smaller than a slope of $\sim 2.5$ which, if there were no ionospheric and tropospheric correction, would likely be expected on the basis of the $\sim 2.5: 1$ ratio of the separations of the two sources from $\mathrm{C} 1$. However, this slope is significantly $(\sim 4 \sigma)$ larger than unity and also larger than the corresponding slope for the data corrected with the JPL model. The JPL model thus seems to provide a better correction for the ionosphere.

An approximate estimate of uncorrected errors due to the ionosphere and troposphere for position estimates using PIM (as in Paper V for IM Peg) and an upper limit of such errors for position estimates using the JPL model (this paper) can be obtained from a close inspection of the declination data

\footnotetext{
10 For simplicity we fit the data via least squares only in the vertical direction. Fitting the data in both directions would not significantly alter our results. Also, since the errors in the vertical direction are similar to each other and the errors in the horizontal direction are similar to each other too, we did not weight the data in the fits and also did not plot the errors in the figures.
}

corrected with PIM (lower right panel of Figure 5). While the $\Delta \delta$ values of $\mathrm{C} 1-2252$ vary over a range of 0.19 mas, the corresponding values of $\mathrm{C} 1-2250$ vary over a range of 0.34 mas, 1.78 times larger, as also indicated by the slope. Therefore, the difference of the variations of 0.15 mas can likely be attributed to effects of the ionosphere left uncorrected by PIM. Consequently, we think that for data corrected with PIM the peak-to-peak contribution to the error of the position difference, $\mathrm{C} 1-2250$, in $\delta$ is approximately \pm 0.08 mas. To be conservative, we take this as a standard error. The corresponding contribution to the error of the position difference, $\mathrm{C} 1-2252$, in $\delta$ approximately scales with the separation and is therefore likely to be about 0.03 mas. The contributions to the errors in $\alpha$ that we estimated from scaling are 0.04 mas and again 0.03 mas for the two position differences, respectively. For data corrected with the JPL model, we considered all these estimates for PIM as still more conservative upper limits for the standard deviations of these errors.

\subsubsection{Total Errors}

Adding in quadrature the errors from noise, deconvolution, structure, and propagation, we obtain estimated standard errors in $\alpha$ of the position differences from B2250+194 for the components $\mathrm{C} 1, \mathrm{C} 2, \mathrm{D} 1, \mathrm{D} 2$, and $\mathrm{J} 1$ of 0.06 mas and for the component Jext of 0.07 mas. The corresponding standard errors in $\delta$ are 0.09 mas and 0.10 mas. The corresponding standard errors of the position differences from B2252+172, in both $\alpha$ and $\delta$, for the components $\mathrm{C} 1, \mathrm{C} 2, \mathrm{D} 1, \mathrm{D} 2$, and $\mathrm{J} 1$ are 0.05 mas and for the component Jext 0.07 mas.

\subsubsection{Comparison with Empirically Determined Errors}

For the position differences of these components from B2250+194, our estimated standard errors are somewhat smaller than the empirically determined standard errors which were mostly between the values of 0.08 and 0.10 mas in both $\alpha$ and $\delta$ for these components (see Table 2). The reason for this is the large variation of some of the data points near epoch 2001 which increased the empirically determined errors. For the corresponding position differences from B2252+172, all for epochs after 2001, these estimated standard errors agree well with the empirically determined standard errors of 0.04-0.06 mas in $\alpha$ and $\delta$, respectively, for the components $\mathrm{C} 1, \mathrm{C} 2, \mathrm{D} 1, \mathrm{D} 2$, and $\mathrm{J} 1$ and of 0.07 mas in $\alpha$ and $\delta$ for the component Jext (see Table 3 ).

\subsection{Analysis of Geodetic Group-delay Observations of $3 C 454.3$ and B2250+194}

The data from two of our sources, 3C 454.3 and B2250+194, observed in many of the geodetic VLBI sessions, each extending over about one day, were analyzed by one of us (L.P.) with the VTD/post-Solve software package. We made a weighted least-squares solution using all available geodetic VLBI observations of 3955 sources, including B2250+194, 3C 454.3, and the 212 "defining" sources from the ICRF catalog (Fey et al. 2009), made at 157 stations from 1979 to 2008 (dubbed solution gpb_2008a). All in all we used a total of 6.5 million determinations of group delay from observations made simultaneously at 8.4 and $2.3 \mathrm{GHz}$. This solution forms our CRF. It is consistent with the ICRF2 (Fey et al. 2009), which, however, does not provide information that we need as described below.

In particular, we estimated the coordinates of B2250+194 and 3C 454.3 for each observing session while forcing the coordinates of each other source to be constant. We also solved 

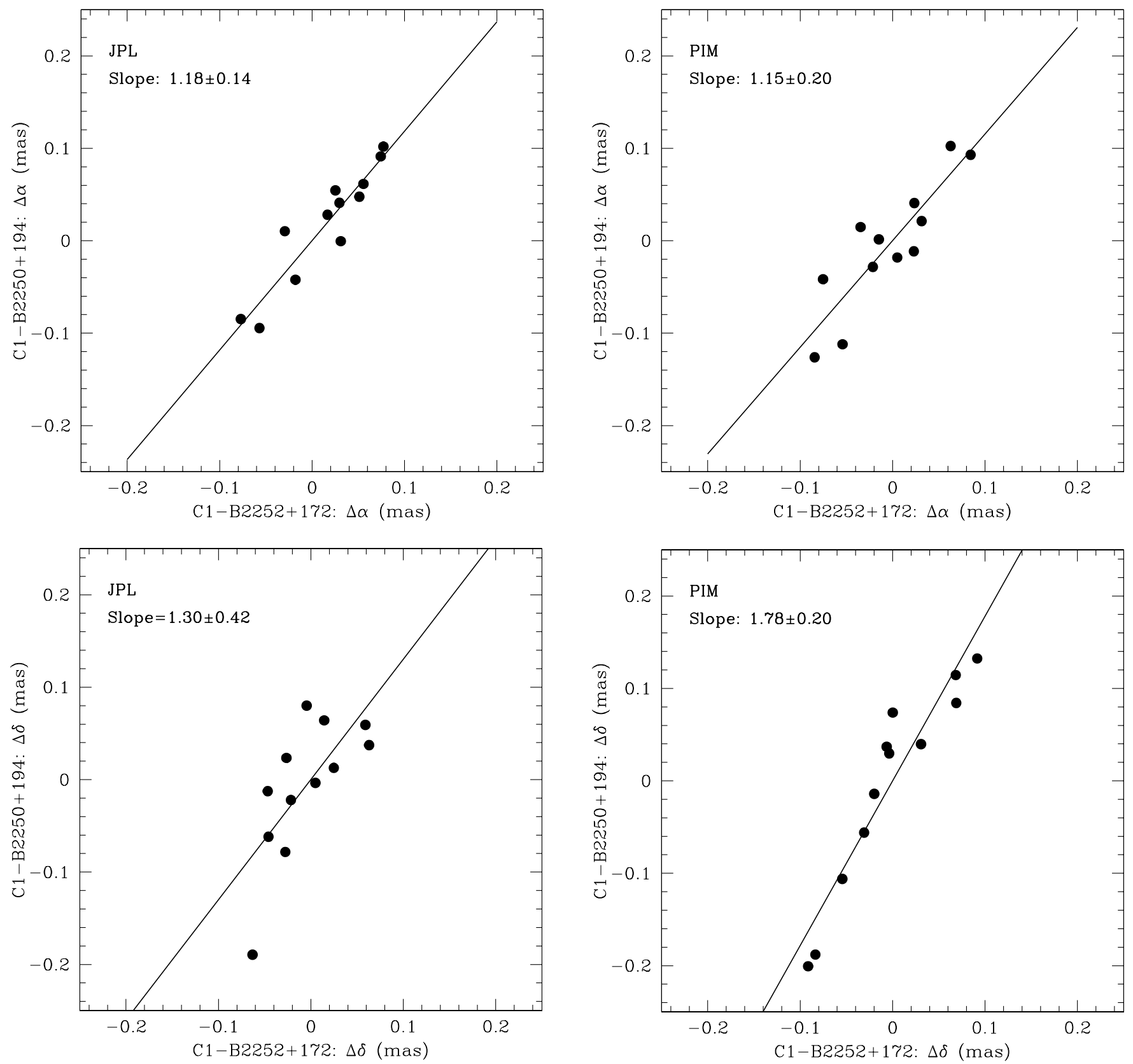

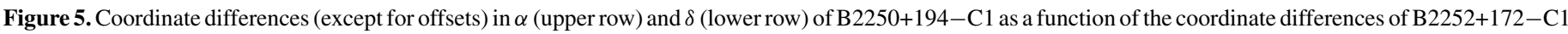

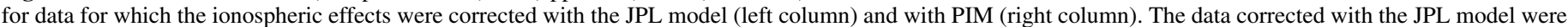

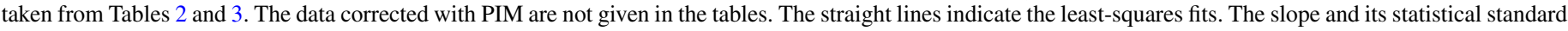
error are given for the fit in each figure.

for the positions and velocities of all stations, for polar motion and UT1 parameters, and their rates of change, for nutation daily offsets at the middle epoch of each session and for numerous other parameters such as those that model clock offsets, atmosphere path delays in the zenith direction, and tilts of the assumed atmosphere axis of symmetry. We imposed the constraint that the net rotation of our estimates of source positions of the 212 defining sources with respect to the positions of these sources in the ICRF catalog be zero. Such a choice for the constraint provides the continuity of our solution to other VLBI solutions, including the ICRF2 solution. For more details, see Petrov et al. (2009).

The individual source positions from this solution were estimated not with respect to a particular reference source, but with respect to the entire ensemble of observed sources. This approach was possible because the geodetic VLBI sessions were designed in such a way that (1) a set of $\sim 100$ core sources and $\sim 20$ core antenna sites ("stations") were common to all sessions, and (2) the resulting estimates of source positions, station positions, and Earth orientation parameters would not be strongly correlated. The presence of sources common to all sessions tended to ensure the consistency of determination of the interferometer orientation with respect to these core sources.

Although our list of sources has 3955 objects, the relative weight of the $\sim 100$ predominant sources ensured that they dominate the ensemble. Therefore, we interpret the estimated positions of B2250+194 and 3C 454.3 as positions almost 


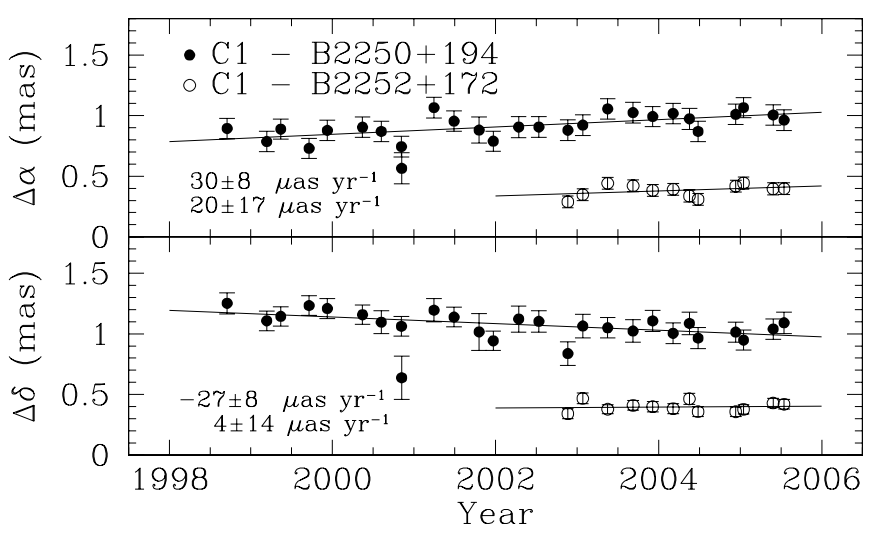

Figure 6. Coordinates of $\mathrm{C} 1$ in $3 \mathrm{C} 454.3$ relative to those of $\mathrm{B} 2250+194$ and $\mathrm{B} 2252+172$ (except for offsets) as a function of time. The values of $\Delta \alpha$ and $\Delta \delta$ are obtained from the entries in Tables 2 and 3. For discussion of apparent partial correlations between the two position-difference data sets, see the text.

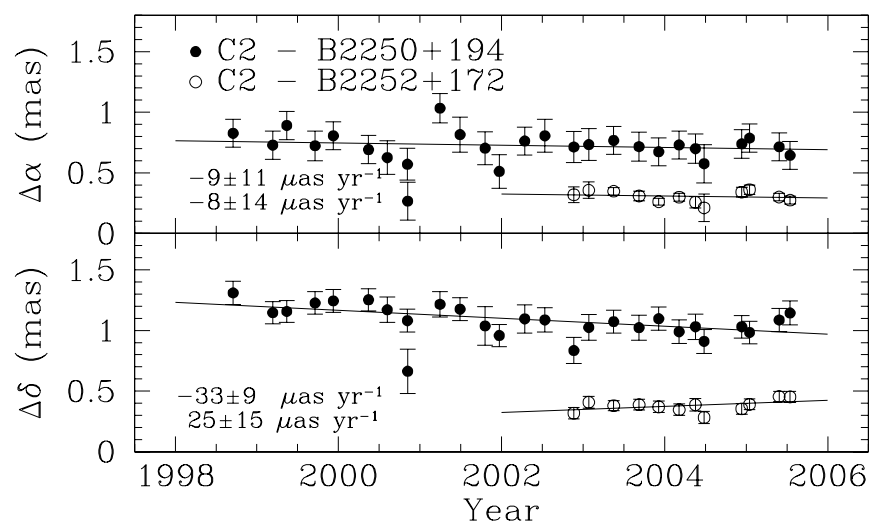

Figure 7. As in Figure 6, but now for $\mathrm{C} 2$ in $3 \mathrm{C} 454.3$.

entirely with respect to the ensemble of the $\sim 100$ predominant extragalactic objects.

For the solution yielding our CRF, each source was assumed to be a point source. However, almost all of these sources exhibit some extended structure at the milliarcsecond scale that may also vary over time. Since these sources were not routinely imaged and hence the group delays not corrected for structure effects, a position determination from geodetic VLBI cannot be identified with respect to a specific fiducial point in the brightness distribution of a source. That fiducial point is therefore in principle unidentified for any source with structure. In general, however, the more compact the source, the closer the estimated position is to the peak in the brightness distribution of the source. The source B2250+194 is sufficiently compact that, for our purposes, the position determined is effectively that of the brightness peak. For 3C 454.3 the fiducial point for each session is less well known because of the complexity of the source structure and its changes with time. Moreover, the fiducial point could change due to changes in the $u-v$ coverage. For these reasons, we do not rely on the $3 \mathrm{C} 454.3$ position from geodetic VLBI observations, but rather use our dedicated VLBI observations and phase-referenced data to identify individual components in the brightness distribution of $3 \mathrm{C} 454.3$ and to determine their positions relative to our two more compact extragalactic sources, B2250+194 and B2252+172, which in turn we tie to the CRF.

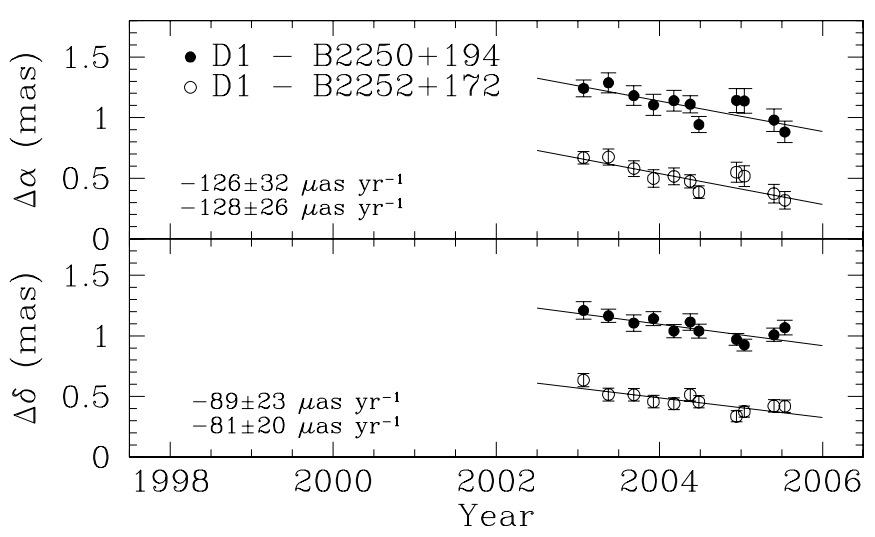

Figure 8. As in Figure 6, but now for D1 in 3C 454.3.

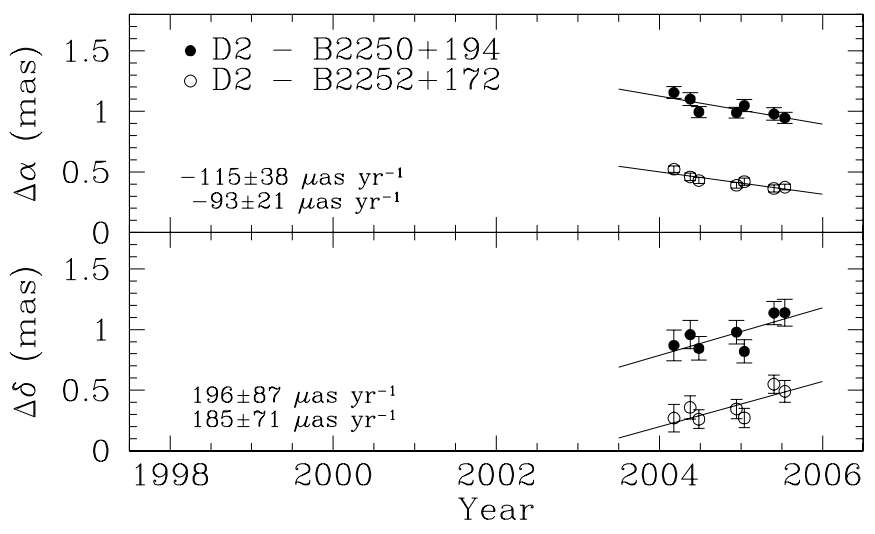

Figure 9. As in Figure 6, but now for D2 in 3C 454.3.

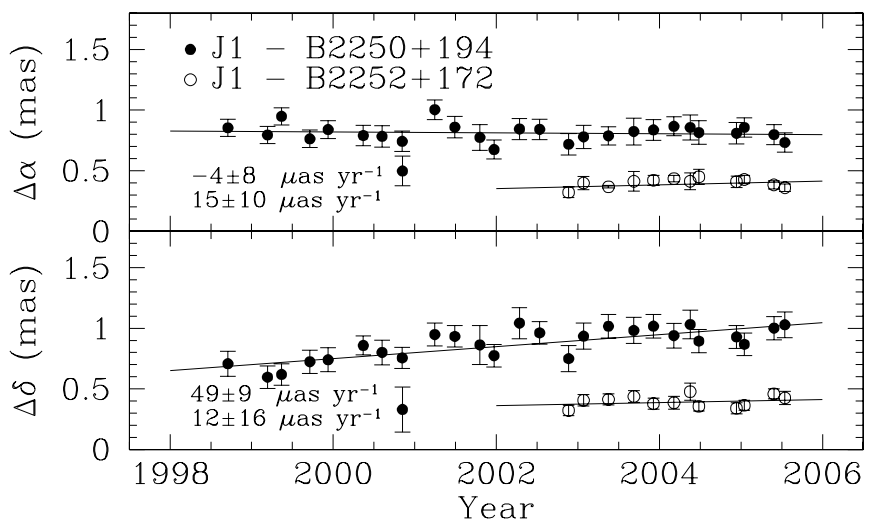

Figure 10. As in Figure 6, but now for $\mathrm{J} 1$ in $3 \mathrm{C} 454.3$.

\section{ASTROMETRIC RESULTS (2): FIT FOR THE POSITION AT EPOCH AND PROPER MOTION}

\subsection{Fit: Components of $3 C 454.3$ Relative to B2250+194 and B2252+172}

We determined the position at epoch and proper motion of each of C1, C2, D1, D2, J1, and Jext relative to the brightness peaks of B2250+194 and B2252+172 with weighted leastsquares fits for $\alpha$ and separately for $\delta$. We list our results together with the weighted linear correlation coefficients and the weighted post-fit rms values (wrms) in Table 4 (solutions nos. 1 and 2). We used the data corrected with the JPL ionosphere model from Tables 2 and 3 . The data and the corresponding lines from the fits (solutions nos. 1 and 2) are plotted in Figures 6-11. Not surprisingly, the smallest wrms values, in both $\alpha$ and $\delta$, were 
Table 4

Relative Positions at Epoch and Proper Motions of the Components of 3C 454.3, B2250+194, and B2252+172a

\begin{tabular}{|c|c|c|c|c|c|c|c|c|c|c|c|c|c|c|}
\hline \multirow{2}{*}{$\begin{array}{l}\text { Component } \\
\text {-reference }\end{array}$} & \multicolumn{3}{|r|}{$\alpha$} & \multirow{2}{*}{ 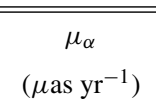 } & \multirow[t]{2}{*}{$\rho_{\alpha}$} & \multirow{2}{*}{$\begin{array}{c}\mathrm{wrms}_{\alpha} \\
(\mu \mathrm{as})\end{array}$} & \multicolumn{3}{|r|}{$\delta$} & \multirow{2}{*}{ 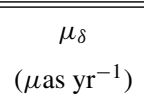 } & \multirow[t]{2}{*}{$\rho_{\delta}$} & \multirow{2}{*}{$\begin{array}{c}\mathrm{wrms}_{\delta} \\
(\mu \mathrm{as})\end{array}$} & \multirow[t]{2}{*}{ Range of Epochs ${ }^{b}$} & \multirow[t]{2}{*}{ Solution No. } \\
\hline & (h) & $(\mathrm{m})$ & (s) & & & & $\left({ }^{\circ}\right)$ & $\left({ }^{\prime}\right)$ & $(")$ & & & & & \\
\hline $\mathrm{C} 1-2250$ & & & $50.3787837(19)$ & $30 \pm 8$ & 0.60 & 93 & -3 & 33 & $41.067505(28)$ & $-27 \pm 8$ & -0.56 & 85 & 1998.71-2005.54 & $1^{\mathrm{c}}$ \\
\hline$C 2-2250$ & & & $50.3787347(27)$ & $-9 \pm 11$ & -0.16 & 119 & -3 & 33 & $41.067591(32)$ & $-33 \pm 9$ & -0.58 & 97 & $1998.71-2005.54$ & $1^{\mathrm{c}}$ \\
\hline D1-2250 & & & $50.3786586(25)$ & $-126 \pm 32$ & -0.79 & 74 & -3 & 33 & $41.067913(23)$ & $-89 \pm 23$ & -0.79 & 52 & 2003.07-2005.54 & $1^{\mathrm{c}}$ \\
\hline D2-2250 & & & $50.3784787(14)$ & $-115 \pm 38$ & -0.80 & 41 & -3 & 33 & $41.067960(42)$ & $196 \pm 87$ & 0.71 & 88 & $2004.18-2005.54$ & $1^{\mathrm{c}}$ \\
\hline $\mathrm{J} 1-2250$ & & & $50.3783932(19)$ & $-4 \pm 8$ & -0.10 & 80 & -3 & 33 & $41.066488(33)$ & $49 \pm 9$ & 0.73 & 96 & $1998.71-2005.54$ & $1^{\mathrm{c}}$ \\
\hline Jext -2250 & & & $50.3783141(24)$ & $-51 \pm 10$ & -0.72 & 103 & -3 & 33 & $41.065042(27)$ & $70 \pm 8$ & 0.87 & 83 & $1998.71-2005.54$ & $1^{\mathrm{c}}$ \\
\hline $\mathrm{C} 1-2252$ & & -01 & $01.8494857(14)$ & $20 \pm 17$ & 0.36 & 45 & -1 & 24 & $31.129358(16)$ & $4 \pm 14$ & 0.09 & 37 & 2002.89-2005.54 & $2^{\mathrm{d}}$ \\
\hline $\mathrm{C} 2-2252$ & & -01 & $01.8495343(10)$ & $-8 \pm 14$ & -0.19 & 35 & -1 & 24 & $31.129424(18)$ & $25 \pm 15$ & 0.46 & 42 & 2002.89-2005.54 & $2^{\mathrm{d}}$ \\
\hline D1-2252 & & -01 & $01.8496108(20)$ & $-128 \pm 26$ & -0.86 & 59 & -1 & 24 & $31.129789(20)$ & $-81 \pm 20$ & -0.80 & 46 & 2003.07-2005.54 & $2^{\mathrm{d}}$ \\
\hline $\mathrm{D} 2-2252$ & & -01 & $01.8497412(08)$ & $-93 \pm 21$ & -0.89 & 23 & -1 & 24 & $31.129750(35)$ & $186 \pm 72$ & 0.76 & 72 & 2004.18-2005.54 & $2^{\mathrm{d}}$ \\
\hline $\mathrm{J} 1-2252$ & & -01 & 01.8498757 (19) & $15 \pm 10$ & 0.43 & 26 & -1 & 24 & $31.128410(19)$ & $12 \pm 16$ & 0.24 & 43 & 2002.89-2005.54 & $2^{\mathrm{d}}$ \\
\hline Jext-2252 & & -01 & 01.8499544 (16) & $-59 \pm 21$ & -0.67 & 55 & -1 & 24 & $31.126911(25)$ & $96 \pm 22$ & 0.81 & 59 & 2002.89-2005.54 & $2^{\mathrm{d}}$ \\
\hline $\mathrm{C} 1-2250 / 2252$ & & & & $20 \pm 13$ & & & & & & $7 \pm 12$ & & & 2002.89-2005.54 & $3^{\mathrm{e}}$ \\
\hline $\mathrm{C} 2-2250 / 2252$ & & & & $-10 \pm 11$ & & & & & & $28 \pm 14$ & & & 2002.89-2005.54 & $3^{\mathrm{e}}$ \\
\hline D $1-2250 / 2252$ & & & & $127 \pm 20$ & & & & & & $-84 \pm 15$ & & & 2002.89-2005.54 & $3^{\mathrm{e}}$ \\
\hline $\mathrm{D} 2-2250 / 2252$ & & & & $-98 \pm 18$ & & & & & & $190 \pm 56$ & & & 2002.89-2005.54 & $3^{\mathrm{e}}$ \\
\hline $\mathrm{J} 1-2250 / 2252$ & & & & $12 \pm 8$ & & & & & & $15 \pm 14$ & & & 2002.89-2005.54 & $3^{\mathrm{e}}$ \\
\hline Jext $-2250 / 2252$ & & & & $-59 \pm 16$ & & & & & & $104 \pm 15$ & & & $2002.89-2005.54$ & $3^{\mathrm{e}}$ \\
\hline $2252-2250$ & 00 & 01 & $52.2282694(6)$ & $-2 \pm 9$ & -0.06 & 22 & -2 & 09 & $09.938073(20)$ & $5 \pm 19$ & 0.08 & 48 & 2002.89-2005.54 & $4^{\mathrm{f}}$ \\
\hline
\end{tabular}

Notes.

${ }^{\text {a }}$ The coordinates and proper motions of the core and jet components of $3 \mathrm{C} 454.3$ for the reference epoch-the midpoint of the GP-B mission-2005 February 1 (2005.08), using VLBI differential observations of $3 \mathrm{C} 454.3$ relative to B2250+194 and B2252+172. The parameters are derived from weighted least-squares linear fits. The uncertainties are standard errors derived from the fit on the basis of the statistical standard errors of the individual measurements added in quadrature to constants so that $\chi_{v}^{2} / v=1$. We searched for any sign that a possible correlation between consecutive data points (see, e.g., Figure 6 ) could render

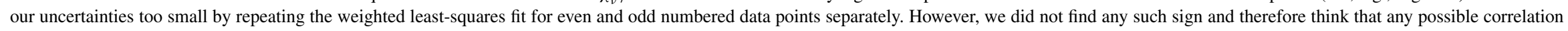

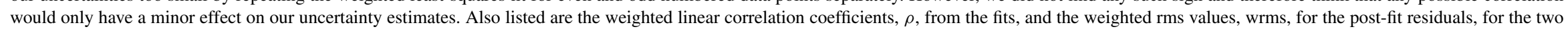
coordinates. All data are corrected for the effects of the ionosphere by using a model implemented in the AIPS software and based on GPS data (JPL, see the text). The earliest date at which the model could be used is 1998 September 17 (1998.71). For D1 and D2 the time range starts with the first epoch at which a component could be identified in the brightness distribution, that is, 2003 January 26 (2003.07) and 2004 March 6 (2004.18), respectively.

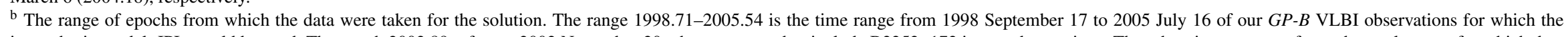
ionospheric model, JPL, could be used. The epoch 2002.89 refers to 2002 November 20 when we started to include B2252+172 in our observations. The other time ranges refer to the total ranges for which data were collected for the respective component and source. The reference epoch 2005.09 is 2005 February 1, the midpoint of the time period through which data were taken on the GP-B spacecraft.

${ }^{c}$ The differences from B2250+194 in the coordinates of the positions and in the corresponding components of the proper motion for each of 3C 454.3's six core and jet components. The time range starts for

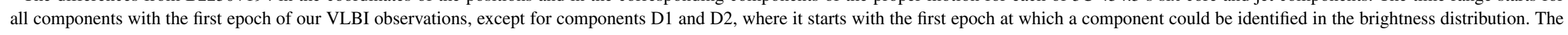
epochs of the time range are 1998 September 17 (1998.71), 2003 January 26 (2003.07), and 2005 July 16 (2005.54).

${ }^{\mathrm{d}}$ As in $\mathrm{c}$ for solution no. 1 but now relative to B2252+172. The time range starts with the date of the first B2252+172 observations, 2002 November 20 (2002.89).

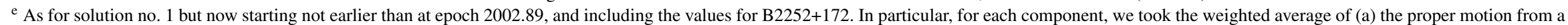

solution (3C 454.3-2250) for the short time range (not listed), and (b) the proper motion of solution no. 2 (3C 454.3-2252). The errors from (a) and (b) were added in quadrature.

${ }^{\mathrm{f}}$ The coordinates of B2252+172 relative to those of B2250+194 and their changes with time. They were derived from the data (2252-C1)-(2250-C1) determined for each epoch. 


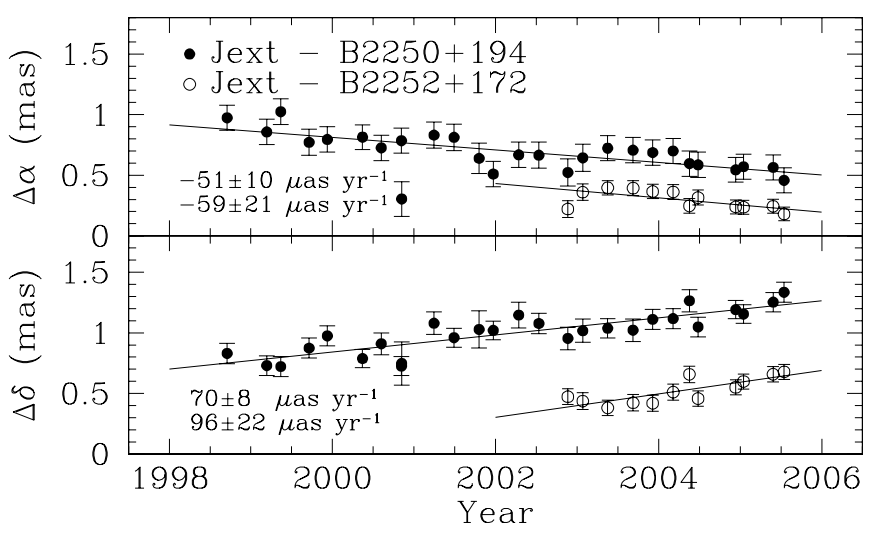

Figure 11. As in Figure 6, but now for Jext in 3C 454.3.

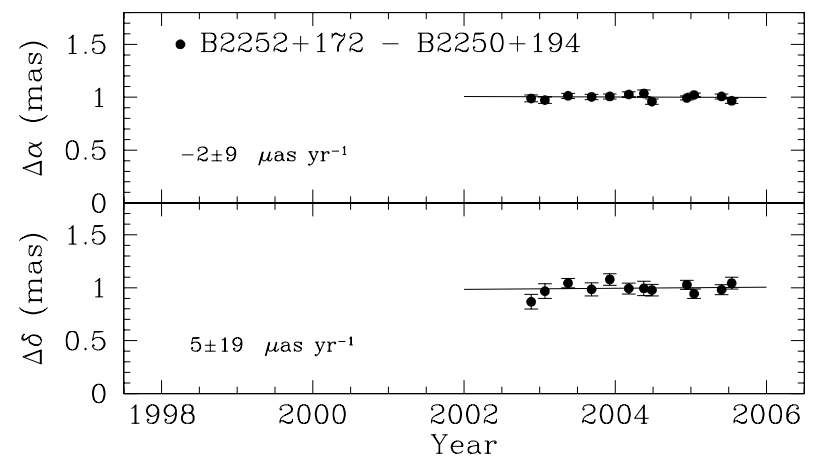

Figure 12. Coordinates of B2252+172 relative to those of B2250+194 (except for an offset in each coordinate) as determined from the differences of the position estimates, $(2252-\mathrm{C} 1)-(2250-\mathrm{C} 1)$ given in Tables 2 and 3.

obtained for the components of $3 \mathrm{C} 454.3$ relative to the close reference B2252+172 (solution no. 2). Our combined propermotion estimates are given as solution no. 3 . They were obtained as a weighted average of proper-motion estimates relative to B2250+194 and B2252+172 for the same (short) time range.

\subsection{Fit: B2252+172 Relative to B2250+194}

The position at epoch and proper motion of B2252+172 relative to $\mathrm{B} 2250+194$ were determined by first differencing the position determinations of $\mathrm{C} 1$ relative to $\mathrm{B} 2250+194$ and $\mathrm{C} 1$ relative to $\mathrm{B} 2252+172$ from Tables 2 and 3 in the sense $(\mathrm{C} 1-2250)-(\mathrm{C} 1-2252) .{ }^{11} \mathrm{We}$ then used weighted least squares to fit a straight line to these differences. We list the results also in Table 4 (solution no. 4) and plot the data with the fit line in Figure 12. The relative proper motion of the two sources is zero within a small portion of $1 \sigma$. The $1 \sigma$ upper limits are 11 and $24 \mu$ as $\mathrm{yr}^{-1}$ in $\alpha$ and $\delta$, respectively.

\subsection{Fit: $3 C 454.3, B 2250+194$, and $B 2252+172$ in the $C R F$}

\subsection{1. $3 C 454.3$}

In Figure 13 we plot the position determinations of 3C 454.3 from geodetic VLBI observations spanning almost 30 years. The statistical standard errors of the coordinates vary widely in part because of the different lengths of time this source was observed in the various sessions, and in part because the sensitivity of the VLBI systems used for the observations generally improved over time.

\footnotetext{
11 Differencing the phase delays for each scan at each epoch would have given us the position of B2252+172 relative to B2250+194 directly for each epoch and likely with a somewhat smaller uncertainty. However, our procedure proved to also give sufficiently accurate results for our purposes.
}

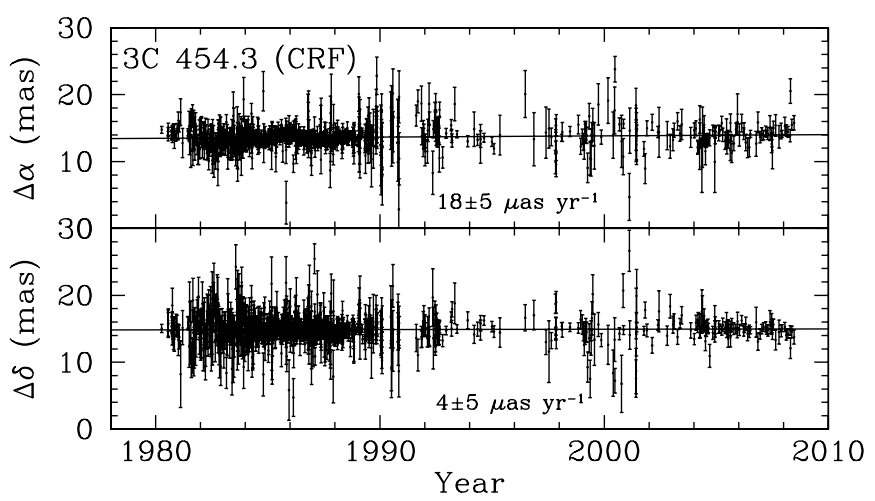

Figure 13. Coordinates of $3 \mathrm{C} 454.3$ as determined from routine geodetic VLBI observations of up to $\sim 4000$ extragalactic sources scattered over the sky. Here and hereafter for plots of coordinates vs. time, the straight lines give the weighted least-squares fit to the data points. The slopes of the lines and their statistical standard errors are indicated. These errors are standard errors adjusted with a constant added in quadrature to the statistical standard errors for each coordinate so that $\chi_{v}^{2} / v=1$ (see the text).

We solved for the position and proper motion of 3C 454.3 by using weighted least-squares fits for $\alpha$ and $\delta$ separately, for the whole time range of observations from 1980 to 2008. Since these weighted least-squares fits to the data gave $\chi_{v}^{2} / v$ larger than unity, we again added a constant in quadrature to the statistical standard errors, separately for $\alpha$ and $\delta$, so as to obtain $\chi_{v}^{2} / v=1$ for each coordinate. The standard errors for the individual data points so determined, together with the fit lines, are plotted in Figure 13 and the results are listed in Table 5 (solution no. 1). In our CRF, the proper-motion component in $\delta$ is zero within $0.8 \sigma$. However, in $\alpha$ it is $18 \pm 5 \mu$ as $\mathrm{yr}^{-1}$, non-zero at a $3.6 \sigma$ significance level. However, since this determination does not refer consistently to any particular component in the brightness distribution of the source, its significance is not clear.

\subsection{2. $B 2250+194$}

The second of our sources observed with geodetic VLBI is B2250+194. About 12 years of such observations yielded determinations of position and proper motion of B2250+194 in the CRF. Via this tie and our phase-delay observations,

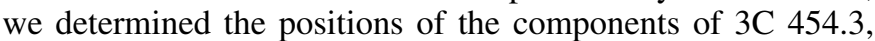
of B2252+172, and of IM Peg in the CRF. For the source B2250+194, the standard errors of the position estimates also vary widely from session to session because of the different spans of time over which observations of this source were spread in the various sessions. As for 3C 454.3, we plot the coordinates for B2250+194 from the different observing sessions and their standard errors, computed as described above, together with the linear fit lines (solution no. 2 in Table 5) for the whole observing period, from 1997 to 2008, in Figure 14. The proper motion is zero within $0.4 \sigma$.

\subsection{3. $B 2252+172$}

Using the position at epoch and proper motion of B2250+194 in the CRF, given in Table 5 (solution no. 2), we also obtain the equivalent values for B2252+172 in the CRF. In particular, we take the values from the above solution no. 2 and add them to the values from our phase-reference observations from Table 4 (solution no. 4) in the sense $2250+(2252-2250)$ and list them also in Table 5 (solution no. 3 ). The proper motion is zero within $0.1 \sigma$.

Owing to a recent VLBA sensitivity upgrade, it became possible now to determine group delays of sources as weak as 10 
Table 5
The Positions at Epoch and Proper Motions in the Celestial Reference Frame $(\mathrm{CRF})^{\mathrm{a}}$

\begin{tabular}{|c|c|c|c|c|c|c|c|c|c|c|c|c|c|c|}
\hline \multirow[t]{2}{*}{ Component-reference } & \multicolumn{3}{|r|}{$\alpha$} & \multirow{2}{*}{$\begin{array}{c}\mu_{\alpha} \\
\left(\mu \mathrm{as} \mathrm{yr}^{-1}\right)\end{array}$} & \multirow[t]{2}{*}{$\rho_{\alpha}$} & \multirow{2}{*}{$\begin{array}{r}\mathrm{wrms}_{\alpha} \\
(\mu \mathrm{as})\end{array}$} & \multicolumn{3}{|r|}{$\delta$} & \multirow{2}{*}{$\begin{array}{c}\mu_{\delta} \\
(\mu \text { asyr })\end{array}$} & \multirow[t]{2}{*}{$\rho_{\delta}$} & \multirow{2}{*}{$\begin{array}{c}\text { wrms } \delta \\
(\mu \text { as })\end{array}$} & \multirow[t]{2}{*}{ Range of Epochs ${ }^{b}$} & \multirow[t]{2}{*}{ Solution No. } \\
\hline & (h) & (m) & (s) & & & & $\left({ }^{\circ}\right)$ & $\left({ }^{\prime}\right)$ & $\left({ }^{\prime \prime}\right)$ & & & & & \\
\hline 3C 454.3 & 22 & 53 & $57.7479664(60)$ & $18 \pm 5$ & 0.11 & 742 & 16 & 08 & $53.560943(80)$ & $4 \pm 5$ & 0.03 & 918 & $1980.28-2008.47$ & 1 \\
\hline B2250+194 & 22 & 53 & $7.3691736(24)$ & $3 \pm 10$ & 0.05 & 179 & 19 & 42 & $34.628786(62)$ & $-7 \pm 20$ & -0.06 & 345 & $1996.01-2008.36$ & 2 \\
\hline B2252+172 & 22 & 54 & $59.5974430(25)$ & $1 \pm 13$ & & & 17 & 33 & $24.690713(65)$ & $-2 \pm 28$ & & & 2002.89-2005.54 & $3^{c}$ \\
\hline C1 (50) & 22 & 53 & $57.7479573(31)$ & $33 \pm 13$ & $\ldots$ & $\ldots$ & 16 & 08 & $53.561281(68)$ & $-35 \pm 21$ & $\ldots$ & $\ldots$ & 1998.71-2005.54 & $4^{\mathrm{d}}$ \\
\hline $\mathrm{C} 2(50)$ & 22 & 53 & $57.7479083(36)$ & $-7 \pm 15$ & $\ldots$ & $\ldots$ & 16 & 08 & $53.561195(70)$ & $-40 \pm 22$ & $\ldots$ & $\ldots$ & 1998.71-2005.54 & $4^{\mathrm{d}}$ \\
\hline D1 (50) & 22 & 53 & $57.7478322(35)$ & $-123 \pm 34$ & $\ldots$ & $\ldots$ & 16 & 08 & $53.560873(66)$ & $-96 \pm 30$ & $\ldots$ & $\ldots$ & 2003.07-2005.54 & $4^{\mathrm{d}}$ \\
\hline D2 (50) & 22 & 53 & $57.7476523(28)$ & $-112 \pm 39$ & $\ldots$ & $\ldots$ & 16 & 08 & $53.560826(75)$ & $188 \pm 89$ & $\ldots$ & $\ldots$ & 2004.18-2005.54 & $4^{\mathrm{d}}$ \\
\hline $\mathrm{J} 1(50)$ & 22 & 53 & $57.7475668(31)$ & $-1 \pm 13$ & $\ldots$ & $\ldots$ & 16 & 08 & $53.562298(70)$ & $42 \pm 22$ & $\ldots$ & $\ldots$ & 1998.71-2005.54 & $4^{\mathrm{d}}$ \\
\hline Jext (50) & 22 & 53 & $57.7474877(34)$ & $-49 \pm 14$ & $\ldots$ & $\ldots$ & 16 & 08 & $53.563744(68)$ & $63 \pm 21$ & $\ldots$ & $\ldots$ & 1998.71-2005.54 & $4^{\mathrm{d}}$ \\
\hline C1 (50/52) & $\ldots$ & $\ldots$ & $\ldots$ & $22 \pm 17$ & $\ldots$ & $\ldots$ & $\ldots$ & $\ldots$ & $\ldots$ & $4 \pm 26$ & $\ldots$ & $\ldots$ & 2002.89-2005.54 & $5^{\mathrm{e}}$ \\
\hline $\mathrm{C} 2(50 / 52)$ & $\ldots$ & $\ldots$ & $\ldots$ & $-8 \pm 15$ & $\ldots$ & $\ldots$ & $\ldots$ & $\ldots$ & $\ldots$ & $25 \pm 27$ & $\ldots$ & $\ldots$ & 2002.89-2005.54 & $5^{\mathrm{e}}$ \\
\hline D1 $(50 / 52)$ & $\ldots$ & $\ldots$ & $\ldots$ & $-125 \pm 23$ & $\ldots$ & $\ldots$ & $\ldots$ & $\ldots$ & $\ldots$ & $-92 \pm 27$ & $\ldots$ & $\ldots$ & 2003.07-2005.54 & $5^{\mathrm{e}}$ \\
\hline D2 (50/52) & $\ldots$ & $\ldots$ & $\ldots$ & $-99 \pm 25$ & $\ldots$ & $\ldots$ & $\ldots$ & $\ldots$ & $\ldots$ & $185 \pm 59$ & $\ldots$ & $\ldots$ & 2004.18-2005.54 & $5^{\mathrm{e}}$ \\
\hline $\mathrm{J} 1(50 / 52)$ & $\ldots$ & $\ldots$ & $\ldots$ & $13 \pm 14$ & $\ldots$ & $\ldots$ & $\ldots$ & $\ldots$ & $\ldots$ & $10 \pm 24$ & $\ldots$ & $\ldots$ & 2002.89-2005.54 & $5^{\mathrm{e}}$ \\
\hline Jext (50/52) & $\ldots$ & $\ldots$ & $\ldots$ & $-58 \pm 25$ & $\ldots$ & $\ldots$ & $\ldots$ & $\ldots$ & $\ldots$ & $100 \pm 26$ & $\ldots$ & $\ldots$ & 2002.89-2005.54 & $5^{\mathrm{e}}$ \\
\hline
\end{tabular}

Notes.

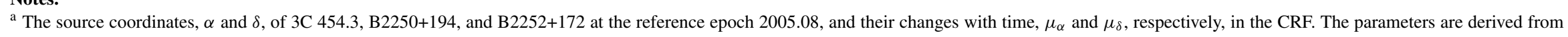
weighted least-squares linear fits. The uncertainties are standard errors derived from the fit on the basis of the statistical standard errors of the individual measurements added in quadrature to constants so that $\chi_{v}^{2} / v=1$. Also listed are the weighted linear correlation coefficients, $\rho$, from the fits, as well as the weighted rms values, wrms, for the post-fit residuals, for the two coordinates.

${ }^{\mathrm{b}}$ The range of epochs as in Table 5 .

${ }^{c}$ The coordinates of B2252+172 and their changes with time derived by adding the solutions from no. 2 to those of no. 4 in Table 4. The errors were added in quadrature.

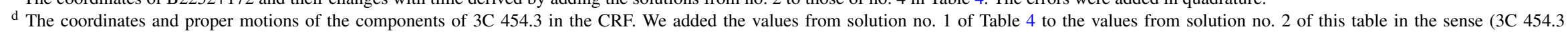
comp. -2250$)+2250$, and added the errors in quadrature.

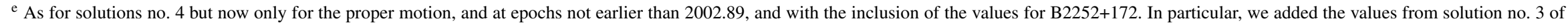
Table 4 to the values from solution no. 2 of this table in the sense (3C 454.3 comp.-2250/2252) +2250 and added the errors in quadrature. 


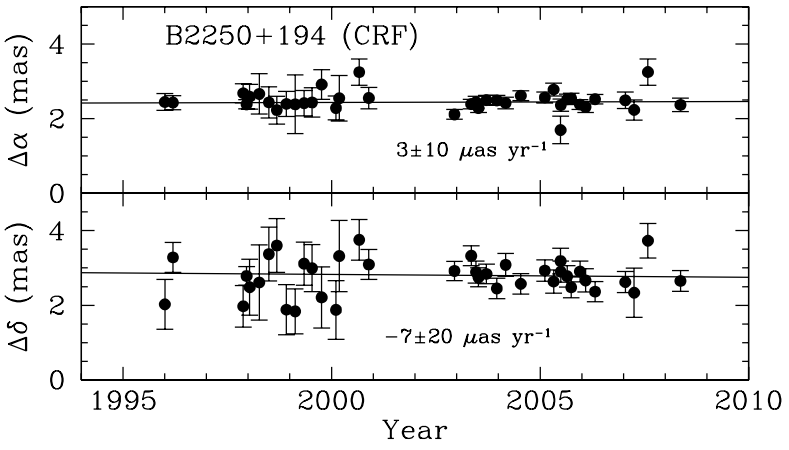

Figure 14. Change in coordinates of B2250+194. Otherwise similar to Figure 13.

mJy. Source B2252+172 was observed in two scans each $320 \mathrm{~s}$ long, with a nine-station VLBA network at epoch 2011 August 14 at $8.4 \mathrm{GHz}$ during a gap in the schedule of target sources in a VLBI survey of bright infrared galaxies (Condon et al. 2011). The source appeared slightly resolved with a correlated flux density in a range of 11-13 mJy at a range of baseline projection lengths of 3-200 megawavelengths. Its group-delay coordinates from that experiment are $22^{\mathrm{h}} 54^{\mathrm{m}} 59.597449 \pm$ $0.000015,+17^{\circ} 33^{\prime} 24^{\prime \prime} 69049 \pm 0.00042$. These coordinates agree with those from Table 4 (solution no. 3 ) within the former's sixfold larger uncertainties. The corresponding proper motion for the 6.6 years between our reference epoch (see Table 4) and the observing date in 2011 is $13 \pm 33 \mu$ as and $-34 \pm 64 \mu$ as, which gives $1 \sigma$ upper limits about threefold larger than those in Table 4.

\subsection{Fit: Components of $3 C 454.3$ in the CRF}

We determined the position at epoch and proper motion of each of C1, C2, D1, D2, J1, and Jext in the CRF by combining the values relative to $\mathrm{B} 2250+194$ and $\mathrm{B} 2252+172$ with the values of the latter sources in the CRF. In particular for the positions, we take the values from Table 4 (solution no. 1) and add them to the values from Table 5 (solution no. 2) in the sense $(3 \mathrm{C} 454.3$ components -2250$)+2250$. For the proper-motion values we include the data involving B2252+172 since they are independent of those involving B2250+194. We thus take all data into account, but do so only for the restricted range of epochs for which we have B2252+172 VLBI observations. In particular, we took the average solution no. 3 from Table 4 and added to it the values of B2250+194 in the CRF by using the solution no. 2 from Table 5 in the sense (3C 454.3 components-2250/2252) + 2250. We list the resulting position-at-epoch and proper-motion values for each component in Table 5 (solution no. 4).

\subsubsection{Position of the Core Component, C1, of $3 C 454.3$}

We now discuss position determinations obtained via our two different astrometric techniques: the position of $3 \mathrm{C} 454.3$ in the CRF obtained from geodetic group-delay observations (solution no. 1, Table 5) and the position of $\mathrm{Cl}$ in the CRF obtained through a combination of geodetic group-delay and our phasedelay observations (solution no. 4, Table 5). We emphasize that these two estimates are not expected to coincide, even in principle, because the former relates to some (ill-defined) average over source structure and the latter to a far better defined component $(\mathrm{C} 1)$ within that structure. The difference between the two position determinations (pure group delay minus combination) is $9.1 \pm 6.8 \mu \mathrm{s}$ in $\alpha$ and $-338 \pm 105 \mu$ as in $\delta$. While the difference in $\alpha$ is only $1.3 \sigma$, the difference in $\delta$ is $3.2 \sigma$, large enough to perhaps be significant.

In this context we compare our pure group-delay position estimate for 3C 454.3 with other such estimates. For instance, the ICRF2 catalog (Fey et al. 2009) provides position estimates of $3 \mathrm{C} 454.3$ and B2250+194 ${ }^{12}$ which are close to ours. It is based on essentially the same set of group delays, the same data editing, and the same software as we used but with a slightly different reduction and estimation model. The differences (ours-ICRF2) are $-16 \pm 62 \mu \mathrm{s}$ and $-0.2 \pm 3.5 \mu \mathrm{s}$ in $\alpha$ and $-4 \pm 970 \mu$ as and $39 \pm 62 \mu$ as in $\delta$, for 3 C 454.3 and B2250+194, respectively. Here we take our estimate of an apparent proper motion in $\alpha$ of $18 \pm 5 \mu \mathrm{as} \mathrm{yr}^{-1}$ into account and propagate our estimate back 11.13 years to their mean epoch of 1993.95. Our estimate of the proper motion in $\delta$ and the corresponding estimates for B2250+194 were small enough so that it was not necessary to consider them. The uncertainties are those from the ICRF2 listings only.

Further, the latest estimate from the USNO CRF solution, crf2009b, ${ }^{13}$ which uses essentially the same data set as we used only extended by another year, is different from ours (in the sense (ours-USNO)) by $-1.7 \pm 6.1 \mu \mathrm{s}$ and $-1.4 \pm 1.9 \mu \mathrm{s}$ in $\alpha$ and $-21 \pm 17 \mu$ as and $52 \pm 31 \mu$ as in $\delta$, for $3 C 454.3$ and B2250+194, respectively. Here again we take our estimate of an apparent proper motion in $\alpha$ for 3C 454.3 into account and propagate our estimate back 17.79 years to their mean epoch of 1987.29. The uncertainty is our error from the proper motion added in quadrature with the USNO position error. For the other differences, the proper-motion estimates did not need to be taken into account. The uncertainties are those from the USNO position estimates only.

Another estimate of the position of 3C 454.3, but not of B2250+194, was made recently with group-delay observations at $24 \mathrm{GHz}$ (Lanyi et al. 2010). The corresponding differences are $-15.6 \pm 8 \mu$ s and $-177 \pm 176 \mu$ as. With our, statistically independent, errors added in quadrature, the difference in $\alpha$ reduces to $1.6 \sigma$ and in $\delta$ to $0.9 \sigma$. We do not consider these differences to be significant. Nevertheless, note that the difference in $\delta$ is in the direction to reduce the difference with our determination for $\mathrm{C} 1$.

Since we compare here results from different catalogs, we point out that they are almost identical in their overall orientations. The differences in these orientations correspond to a level of only several tens of microarcseconds, which is negligible for our purposes.

To summarize: First there is a $-338 \mu$ as $(3.2 \sigma)$ difference in $\delta$ between our pure group-delay position of $3 \mathrm{C} 454.3$ and the combined group-delay phase-delay position of $\mathrm{C} 1$. Second, our pure group-delay position determinations for 3C 454.3 and, for comparison, also for $\mathrm{B} 2250+194$ agree within $<1.7 \sigma$ with the USNO and ICRF2 position determinations. Third, the position of $3 \mathrm{C} 454.3$ at $24 \mathrm{GHz}$ in $\delta$, while its error is large, cuts the above $3 \mathrm{C} 454.3 / \mathrm{C} 1$ discrepancy in half. We discuss these results below in Section 8.

\subsubsection{Limit on the Proper Motion of the Core Component C1 of 3 C 454.3}

Table 4 (solution no. 1) shows that from 1998 to 2005 the proper motion of $\mathrm{C} 1$ relative to $\mathrm{B} 2250+194$ is in the southeast direction at a significance level of $3.8 \sigma$ and $3.4 \sigma$ in $\alpha$ and $\delta$,

\footnotetext{
12 This source is incorrectly referred to as B $2250+190$ in the ICRF2 catalog.

$13 \mathrm{http}: / /$ rorf.usno.navy.mil/vlbi/
} 
respectively. In contrast, from 2002 to 2005 the motion of $\mathrm{C} 1$ relative to either B2250+194 (solution not listed) or B2252+172 (solution no. 2) is smaller, and with an uncertainty corresponding to $<1.2 \sigma$, not significant, although the errors are larger. In any case, it appears that either B2250+194 moved to the northwest or $\mathrm{C} 1$ indeed moved to the southeast, particularly during the time from 1998 to 2002.

The cause of this apparent motion is not clear. Is it possible that the brightness peak of B2250+194 belongs to a jet that moved northwestward during the early time period? Figure 3 shows that B2250+194 is elongated to the northwest and also slightly to the south, with the brightness peak located near the center of its curved structure. If a supermassive black hole were located at the southern end of the structure, then the brightness peak would likely belong to a jet component moving away from the black hole, in this case to the northwest. The geodetic observations that determined the proper motion of B2250+194 in the CRF show an insignificant northward motion

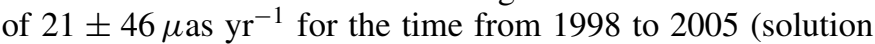
not listed). The observations over the longer period from 1996 to 2008 are collectively more sensitive, but do not indicate any motion to the northwest. Moreover, the geodetic observations of $3 \mathrm{C} 454.3$ itself are too insensitive for a useful proper-motion determination for the period from 1998 to 2005 .

A useful way to test this jet hypothesis would be to determine the position of the brightness peak of B2250+194 at other frequencies (see also Kovalev et al. 2008). The location of the peak at the highest radio frequencies is expected to be close to the source's core and the putative black hole there. For the similarly compact and elongated source, M81*, in the center of the nearby galaxy M81, this method did lead to the confirmation of the approximate location of the core of M81*. This location was earlier determined as the most stationary in the varying brightness distribution of M81* relative to another source (the shell center of SN 1993J) in the same galaxy (Bietenholz et al. 2001; see also Bietenholz et al. 2004). However, our observations at 5 and $15 \mathrm{GHz}$ were not planned for highprecision astrometry and could not be used for this purpose. Our 5 and $15 \mathrm{GHz}$ images appear to be, respectively, just larger and smaller versions of our $8.4 \mathrm{GHz}$ images with the brightness peak remaining in the center of the image (Paper II), without giving any hint as to whether the core may be located in the south of the structure.

As reported in Paper II, B2250+194 undergoes slight structure changes. When the source is modeled with an elliptical Gaussian, the major axis of the Gaussian varies between $\sim 0.6$ and $\sim 0.8$ mas along a position angle of $-11^{\circ}$ over the time from 1997 to 2005 . These structural changes could at least partly account for the nominal proper motion of $\mathrm{C} 1$ relative to $\mathrm{B} 2250+194$.

What is the $1 \sigma$ upper limit on the proper motion of $\mathrm{C} 1$ ? From the point of view of our IM Peg VLBI observations from 1997 to 2005 for which we use C1 as a reference, the degree of stationarity for that period is relevant. For our longest period for the data corrected with JPL, from 1998 to 2005, we obtain for $\mathrm{C} 1$ a $1 \sigma$ proper-motion limit relative to $\mathrm{B} 2250+194$ of 38 and $35 \mu$ as $\mathrm{yr}^{-1}$ in $\alpha$ and $\delta$, respectively (solution no. 1, Table 4). The equivalent upper limit of the proper motion in the CRF over that period is 46 and $56 \mu \mathrm{as} \mathrm{yr}^{-1}$ in $\alpha$ and $\delta$, respectively (solution no. 4, Table 5). For $G P-B$ (see Section 1), this is our fundamental result on the level of stationarity of the chosen single reference point, $\mathrm{C} 1$, relative to the distant universe.

For the shorter period, from 2002 to 2005, no significant proper motion for $\mathrm{C} 1$ was found above the $1.5 \sigma$ level, relative neither to B2250+194 nor to B2252+172, and not within the CRF. For this period we obtain smaller limits that are interesting from an astrophysical point of view. $\mathrm{C} 1$ is stationary relative to the combination of the two reference sources within a $1 \sigma$ upper limit of 33 and $19 \mu \mathrm{as} \mathrm{yr}^{-1}$ (solution no. 3 in Table 5) and in

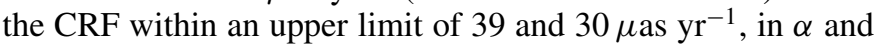
$\delta$, respectively (solution no. 5 , Table 5).

\subsubsection{Motion of the Jet Components of $3 C 454.3$}

What are the proper motions of the components other than $\mathrm{C} 1$ relative to the two reference sources and in the CRF? Are they significantly different from that of $\mathrm{C} 1$ and, moreover, can significant motion be detected of any of them relative to the distant universe? In Paper II we showed that, on average, C2, $\mathrm{D} 1, \mathrm{D} 2, \mathrm{~J} 1$, and Jext are all moving away from $\mathrm{C} 1$. Their relative speeds are not necessarily constant over the more than seven years of our observations. Most dramatically, J1 moves at about twice its average speed until 2002 and then slows down to almost zero velocity thereafter (Paper II).

In Table 4 we list the proper motions of these components relative to each of the two reference sources and to both combined, and in Table 5 to the CRF. For C2 we find motion with a larger significance than that for $\mathrm{C} 1$ only relative to B2252+172 (solution no. 2) and to both reference sources combined $(2.0 \sigma$ in $\delta$, solution no. 3). However, we do not regard the significance large enough to consider the motion real. Relative to B2250+194 and to the CRF, C2 is stationary at about the same significance level as C1. For J1 a clear average northward motion of $49 \pm 9 \mu$ as $\mathrm{yr}^{-1}(5.4 \sigma)$ relative to B2250+194 is observed between 1998 and 2005 (solution no. 1). The significance, however, decreases to only $1.9 \sigma$, due to larger uncertainties, when the motion is measured in the CRF (solution no. 4), comparable to the significance of the motions in the CRF of each, $\mathrm{C} 1$ and $\mathrm{C} 2$. The motion itself decreases to (almost) zero within the errors for the period from 2002 to 2005 relative to either B2250+194 (solution not listed) or B2252+172 (solution no. 2) or both (solution no. 3) or to the CRF (solution no. 5) consistent with J1's motion relative to $\mathrm{C} 1$. The largest and/or most significant motions, relative to either of the two reference sources or both combined or in the CRF were found for D1, D2, and Jext, with, for instance, D2 having a speed in the CRF of $-99 \pm 25$ and $185 \pm 59 \mu \mathrm{as} \mathrm{yr}^{-1}$ in $\alpha$ and $\delta$, respectively, for the short period from 2004 to 2005 (solution no. 5).

To summarize, for the long period from 1998 to $2005, \mathrm{C} 1$ and $\mathrm{C} 2$ are stationary within the same small bounds relative to $\mathrm{B} 2250+194$ and are joined by $\mathrm{J} 1$ when the stationarity is measured (less accurately) in the CRF. The other components are stationary only within larger bounds or move significantly. For the short period from 2002 to $2005, \mathrm{C} 1$ and $\mathrm{J} 1$ are stationary within the same small bounds relative to $\mathrm{B} 2252+172$ and to both reference sources combined, and are joined by $\mathrm{C} 2$ when the stationarity is measured in the CRF. Again, the other components are stationary only within larger bounds or show significant proper motion. The largest significant proper motion was found for D2.

\section{ASTROMETRIC RESULTS (3): ANALYSIS OF OTHER MOTIONS}

\subsection{Fit: Parallax, Proper Acceleration, and Orbital Motion of the Core Component, C1, of 3C 454.3}

Since our computation of the motion of the guide star IM Peg includes solutions for parallax and proper acceleration, to help 
Table 6

Proper Motion, Parallax, and Proper Acceleration ${ }^{\mathrm{a}}$

\begin{tabular}{lccccc}
\hline \hline Source-reference & $\begin{array}{c}\mu_{\alpha} \\
\left(\mu \mathrm{as} \mathrm{yr}^{-1}\right)\end{array}$ & $\begin{array}{c}\mu_{\delta} \\
\left(\mu \mathrm{as} \mathrm{yr}^{-1}\right)\end{array}$ & $\begin{array}{c}\pi \\
(\mu \mathrm{as})\end{array}$ & $\begin{array}{c}\dot{\mu}_{\alpha} \\
\left(\mu \mathrm{as} \mathrm{yr}^{-2}\right)\end{array}$ & $\begin{array}{c}\dot{\mu}_{\delta} \\
\left(\mu \mathrm{as} \mathrm{yr}^{-2}\right)\end{array}$ \\
\hline $\mathrm{C} 1-2250$ & $29 \pm 8$ & $-27 \pm 8$ & $40 \pm 20$ & $\ldots$ & $\ldots$ \\
$\mathrm{C} 1-2252$ & $21 \pm 18$ & $4 \pm 14$ & $-2 \pm 17$ & $\ldots$ & $\ldots$ \\
$\mathrm{C} 1-2250$ & $33 \pm 26$ & $11 \pm 27$ & $38 \pm 20$ & $2 \pm 9$ & $13 \pm 9$ \\
$\mathrm{C} 1-2252$ & $3 \pm 44$ & $13 \pm 37$ & $-2 \pm 18$ & $-21 \pm 47$ & $11 \pm 40$ \\
\hline
\end{tabular}

Notes. ${ }^{\text {a }}$ Parameter estimates for component $\mathrm{C} 1$ of $3 \mathrm{C} 454.3$ relative to B2250+194 and, separately, to B2252+172. Uncertainties are statistical standard errors derived from the weighted least-squares fit, scaled to $\chi_{v}^{2} / v=1$. The reference epoch is 2005 February 1.

us put limits on certain systematic errors we also solve for these parameters for $\mathrm{C} 1$. In Table 6 we list the proper motion and the parallax, $\pi$, obtained for $\mathrm{C} 1$ relative to $\mathrm{B} 2250+194$ and separately to $\mathrm{B} 2252+172$ by assuming that both of the reference sources are infinitely distant from Earth. In addition, we solve for the acceleration components, $\dot{\mu}_{\alpha}$ and $\dot{\mu}_{\delta}$, for $\mathrm{C} 1$ and also list these results in Table 6.

Our two most accurate solutions for parallax (relative to $\mathrm{B} 2252+172$ ), unsurprisingly, are zero (to within $0.1 \sigma$ ), with the upper limit being $\sim 20 \mu$ as, corresponding to a distance, $D$, of $>50 \mathrm{kpc}$. Although other data sets might well yield more accurate parallax measurements, or bounds thereon, ours is one of the most, if not the most, accurate obtained so far, given the assumption that the reference sources are sufficiently distant to have negligible parallax. The proper acceleration of $\mathrm{C} 1$ relative to B2250+194 is within $1.4 \sigma$ of zero and is not significant.

We also extended the number of free parameters still further and included a fit to orbital parameters corresponding to the period of the IM Peg binary system of 24.6 days, since such a fit is used in our analysis of IM Peg (Paper V). We found no indication of such orbital motion of $\mathrm{C} 1$, with each of the orbital parameters being zero within $1 \sigma$.

\subsection{Nonlinear Motion of the Core Component, C1, of 3C 454.3 on the Sky?}

Is there significant motion of the core component, $\mathrm{C} 1$, of 3C 454.3 on the sky that departs from the (linear) proper motion inherent in our fit models? Inspecting Figure 6, we see that the motion of $\mathrm{C} 1$ relative to $\mathrm{B} 2250+194$ is somewhat correlated with $\mathrm{C} 1$ 's motion relative to $\mathrm{B} 2252+172$ in both $\alpha$ and $\delta$. To investigate possible nonlinear, or in general, any unmodeled motion in more detail we first plot the coordinates of B2252+172 relative to those of $\mathrm{B} 2250+194$ in Figure 15 . We are using only data corrected for ionospheric effects with the JPL model since this model seems superior to the PIM model and since we are only using data taken during the period of our B2252+172 observations for which corrections with the JPL model were available, i.e., from 2002 to 2005 . The plot shows quasi-random motion with wrms values of $23 \mu$ as in $\alpha$ and $51 \mu$ as in $\delta$. In Figure 16 (upper left panel), we plot the positions of $\mathrm{C} 1$ relative to those of $\mathrm{B} 2250+194$ and $\mathrm{B} 2252+172$. It is apparent that the positions of $\mathrm{C} 1$ are covering a larger area than the positions of $\mathrm{B} 2252+172$ in the previous figure, mainly because of a larger scatter along $\alpha$. The peak-to-peak variations are 2.5 times larger in $\alpha$ but only 1.2 times larger in $\delta$ than in the preceding figures. Also, the positions of $\mathrm{C} 1$ relative to those of $\mathrm{B} 2250+194$ resemble the positions of $\mathrm{C} 1$ relative to those of $\mathrm{B} 2252+172$. These two indications may be evidence for $\mathrm{C} 1$

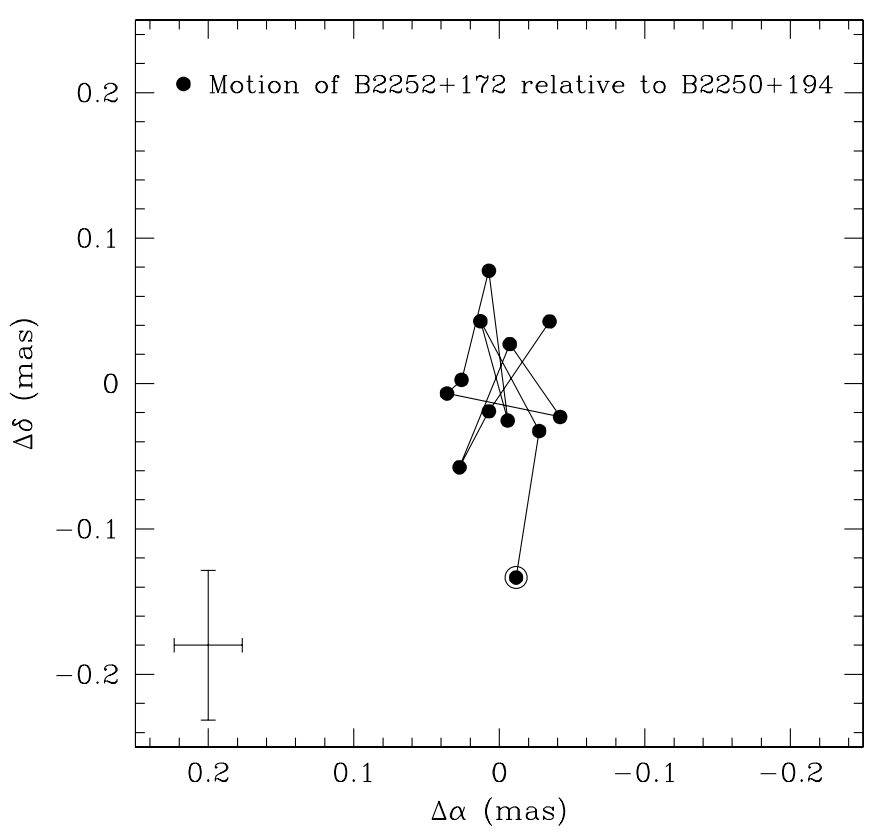

Figure 15. Coordinate determinations, except for an offset, of B2252+172 relative to those of $\mathrm{B} 2250+194$ obtained by taking the difference of the values in Tables 2 and 3 for each epoch in the sense $(\mathrm{C} 1-2250)-(\mathrm{C} 1-2252)$. Errors are left unplotted for clarity but can be computed from the errors given in the above tables. The data point for the first epoch, 2002 November 20, is indicated by a circle around the black dot. The cross in the lower left indicates the weighted rms of the scatter of the data points in the figure, of $23 \mu$ as in $\alpha$ and $51 \mu$ as in $\delta$.

apparently moving on the sky along the east-west axis above the noise level which we adopt to be the wrms values from the B2252+172 versus B2250+194 plot and indicate by the cross in the lower left corner of the figure. In the lower left panel we plot the mean of each pair of position determinations (with $\sqrt{2}$ smaller cross bars) to display this apparent motion more clearly. The plotted motion is confined to an area not larger than $\sim 0.2 \times$ $0.2 \mathrm{mas}^{2}$, a small portion of the beam area but larger than the area of "jittery" motion in Figure 15. Any east-west jittery motion of $\mathrm{C} 1$ is consistent with our simulations (Paper II) of how C1 moves east-west relative to the larger core region structure and relative to the $43 \mathrm{GHz}$ core located $0.18 \pm 0.06$ mas east of $\mathrm{C} 1$. This east-west motion simulated for $\mathrm{C} 1$ is confined to within 0.12 mas. Our finding of possible motion within 0.2 mas is less precise than the result from Paper II, but in principle more accurate since it is measured relative to physically unrelated sources nearby on the sky.

For comparison we show the equivalent motions of the components D1 and Jext in the middle and right columns of Figure 16. As expected from our solution for the proper motion, D1's motion has a linear component to the west-southwest and Jext's toward the northwest.

\section{DISCUSSION}

\subsection{Considerations for Geodetic VLBI with Group Delays}

By comparing the positions of $\mathrm{C} 1$ and 3C 454.3, both in the CRF, we found a possibly significant difference of $-338 \pm$ $105 \mu$ as $(3.2 \sigma)$ in $\delta$ while the difference in $\alpha$ is not significant. To repeat, the position of $\mathrm{C} 1$ in the CRF was determined from the position of $\mathrm{B} 2250+194$ in the $\mathrm{CRF}$ by the addition of the position of $\mathrm{C} 1$ relative to that of $\mathrm{B} 2250+194$. Both the positions of 3C 454.3 and B2250+194 in the CRF were determined from geodetic VLBI group delays while the relative position 

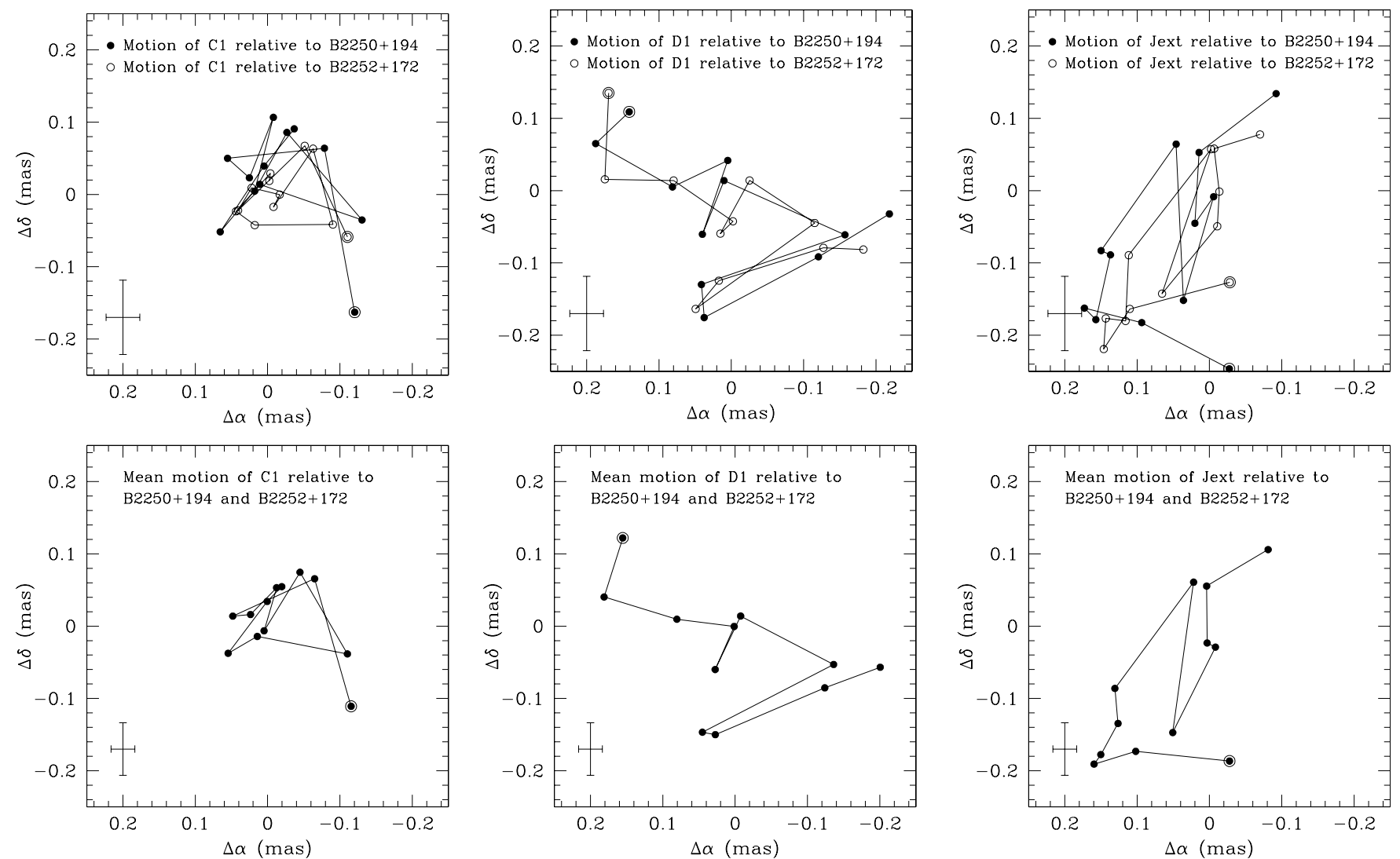

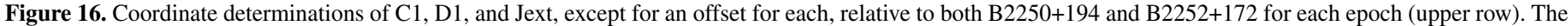

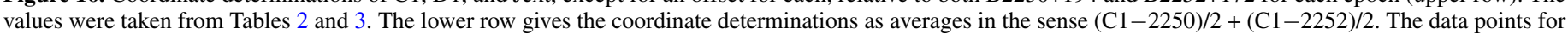

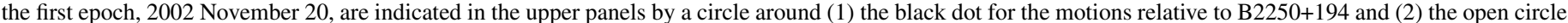

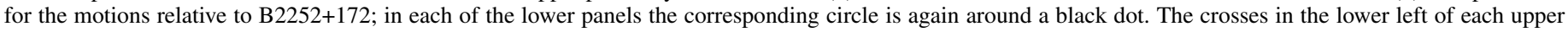

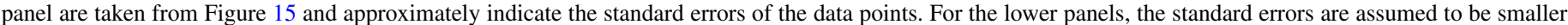
by $\sqrt{2}$.

(C1-2250) was determined from our differential VLBI phase delays. In contrast to the latter, the group-delay observations were not corrected for structure effects. While such effects can be assumed to be insignificant for B2250+194, they may well be significant for $3 \mathrm{C}$ 454.3. Therefore, in contrast to that for B2250+194, the position solution for 3C 454.3 does not refer to a particular reference point in the source's brightness distribution. The solution should therefore be influenced by the structure of the source and its changes with time and frequency. In particular, a shift of the position of the core with decreasing frequency away from the foot of the jet but along the jet axis (see, e.g., Sokolovsky et al. 2011) could influence the results from group delays and phase delays differently.

The source is largely oriented east-west in its brightest part, namely the core region, and oriented toward the northwest in its low-luminosity 10 mas long jet. On first sight one might therefore expect a larger discrepancy in $\alpha$ (see also Porcas 2009) and a smaller one, if any, in $\delta$ and then with a shift to the north, not as observed to the south. If the 10 mas jet caused the discrepancy, it would be via some peculiar influence. The group-delay position determined in 2007 at $24 \mathrm{GHz}$ (Lanyi et al. 2010) indeed diminishes the $\delta$ discrepancy in the position of $\mathrm{C} 1$ by about half although the uncertainty of the $24 \mathrm{GHz}$ position is large. In these observations, 3C 454.3 appears more compact than it does at $8.4 \mathrm{GHz}$. The most easterly component at $24 \mathrm{GHz}$ appears to be pointlike and dominates the image. Low-brightness extended features appear up to 3 mas toward the west but the 10 mas jet is not visible (Charlot et al. 2010) and should therefore have essentially no influence on the position determination. In this context, Martí-Vidal et al. (2008) have also found apparently significant discrepancies between source positions determined from geodetic VLBI group delays without structure corrections and those determined from differential VLBI phase delays with structure corrections. All said, the cause of the discrepancy is not understood.

\subsection{Astrophysical Implications}

\subsubsection{Limit on the Proper Motion of the Core of $3 C 454.3$ and the Proper Motions of the Jet Components}

Since the component $\mathrm{C} 1$ is as close to stationary in the CRF as is any of our other five components of 3C 454.3, and could be closely related to the easternmost compact flat-spectrum component in $43 \mathrm{GHz}$ images (Paper II), this component is likely the closest in our images to the expected supermassive black hole, and therefore very near the center of mass of the quasar. The radio emission probably originates close to the foot of the jet in the vicinity of this putative black hole. The jet components C2, D1, D2, J1, and Jext are all moving away from C1 (Paper II). The motions of D1, D2, and Jext are also significant in the CRF at our sensitivity levels. The proper-motion values of all six components of 3C 454.3 from Table 4 (solution no. 5) were converted to apparent velocities and are listed in Table 7. Our $1 \sigma$ upper limit on the proper motion of $\mathrm{C} 1$ in the CRF of 39 
Table 7

Velocity of the Components of 3C 454.3 in the CRF

\begin{tabular}{lrcc}
\hline \hline Component & \multicolumn{1}{c}{$v_{\alpha} / c$} & $v_{\delta} / c$ & Range of Epochs $^{\mathrm{b}}$ \\
\hline $\mathrm{C} 1$ & $0.6 \pm 0.4$ & $0.1 \pm 0.7$ & $2002.89-2005.54$ \\
$\mathrm{C} 2$ & $-0.2 \pm 0.4$ & $0.6 \pm 0.7$ & $2002.89-2005.54$ \\
$\mathrm{D} 1$ & $-3.2 \pm 0.6$ & $2.3 \pm 0.7$ & $2003.07-2005.54$ \\
D2 & $2.5 \pm 0.6$ & $4.7 \pm 1.5$ & $2004.18-2005.54$ \\
J1 & $0.3 \pm 0.4$ & $0.3 \pm 0.6$ & $2002.89-2005.54$ \\
Jext & $-1.5 \pm 0.6$ & $2.5 \pm 0.7$ & $2002.89-2005.54$ \\
\hline
\end{tabular}

\section{Notes.}

a Velocity in $\alpha$ and $\delta$ in units of the speed of light for angular velocities in Table 5 (solution no. 5). The velocities were computed for an angular diameter distance of 3C 454.3 of $1.6 \mathrm{Gpc}$.

b Same as in Table 4.

and $30 \mu$ as $\mathrm{yr}^{-1}$ in $\alpha$ and $\delta$, respectively, corresponds to a limit of 1.0 and $0.8 c$. The speeds of the jet components in the CRF can be faster and superluminal; for instance, for D2 the speed is $\sim 5 c$.

Similar characteristics are displayed by the superluminal quasar, 3C 345. The core was found to be stationary relative to the physically unrelated quasar, NRAO 512, within $0.4 c$ in the east-west direction while the jet components moved away from the core in this direction at up to $9 c$ (Bartel et al. 1986). ${ }^{14}$ Since in each of these two cases the core is compact and has an approximately flat spectrum in the $\mathrm{GHz}$ frequency range, we conclude that compactness of the component and flatness of the spectrum are indeed, as generally assumed, indicative of the nearby presence of the gravitational center of the quasar.

Component $\mathrm{J} 1$ is special partly because the limit on its speed over the short time interval from 2002 to 2005 is subluminal and partly because its speed was likely much larger at earlier times. Pauliny-Toth (1998) found from VLBI observations at $11 \mathrm{GHz}$ that a component, dubbed "A," moved away from the core toward the northwest from a distance of $\sim 2.8$ mas in 1984 to a distance of $\sim 5.0$ mas in 1992 with a speed averaging $25 c$, but varying from $15 c$ between 1984 and 1985 to $30 c$ between 1985 and 1989 and $20 c$ between 1989 and 1992. This component is likely to be our J1. Jorstad et al. (2005) found that by 2001 this component, dubbed " $D$ " in their paper, has possibly decelerated considerably to $6 c$. The strong deceleration can also be seen in our data from 1998 onward such that by 2002-2005 the component remained stationary within our subluminal limits (see Table 7).

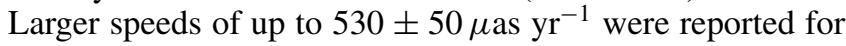
jet components that could be discerned with higher angular resolution at $43 \mathrm{GHz}$ in the $\mathrm{C} 1$ and $\mathrm{C} 2$ area (Jorstad et al. 2001). These authors also speculated on whether perhaps the $43 \mathrm{GHz}$ core could have moved to the northeast by 0.1 mas in $\alpha$ and 0.2 mas in $\delta$ between 1997.6 and 1998.2. While this time range is just before the start of our VLBI observations, our propermotion measurements limit any such motion for later times.

Recently, an exceptionally bright optical outburst was detected in 3C 454.3 (Villata et al. 2006; Vercellone et al. 2010) reaching a maximum in spring 2005 . It was accompanied by an increase of radio emission at $43 \mathrm{GHz}$ from the core that started in early 2005 and reached a maximum in 2005 September. If the outburst is associated with activity of the core, perhaps with the ejection of a new jet component, then the position of $\mathrm{C} 1$ may be expected to be affected. However, inspection of our graphs of the temporal changes in the position of $\mathrm{C} 1$ does not show

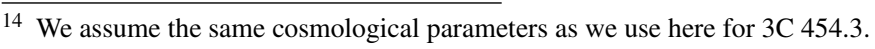

any indication of a possible emerging jet component near $\mathrm{C} 1$, a result not surprising given the 2005 mid-July end of our data set.

To repeat, any transverse linear motion on the sky found for the core of $3 \mathrm{C} 454.3$ has a speed $\leqslant 1.0 \mathrm{c}$. This limit is almost as low in magnitude as the radial velocity of the quasar, computed from its redshift. Transverse velocities comparable in magnitude to the redshift velocities are not expected for quasars for a cosmological model where the dominant motion is the redshift velocity due to the expansion of the universe. Any transverse velocity for the cores of quasars should be at least two orders of magnitude smaller than $c$ based on our knowledge of peculiar motions of galaxies and galaxy clusters. The effect of acceleration of the solar-system barycenter toward the galactic center is also expected to be relatively small. However, it is plausible that a non-zero mean proper motion with respect to the CRF of physically unrelated sources that are separated by less than, say, a radian on the sky is significant because, in effect, we construct the CRF using the approximation that the position of the currently estimated solar-system barycenter is an inertial reference. Fortunately, the estimated acceleration of that barycenter toward the Galactic center has a relatively small effect. At its maximum value (approximately applicable for our

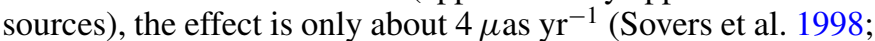
Titov 2010) and has recently been reported to be observed (Titov et al. 2011).

Moreover, any unexpectedly large acceleration of the solarsystem barycenter is less likely, given the study by Zakamska \& Tremaine (2005), who find that pulsar timing data (from both single and binary pulsars) are inconsistent with any unmodeled accelerations of the solar-system barycenter greater than $\sim 4 \times 10^{-9} \mathrm{~cm} \mathrm{~s}^{-2}$, which is only about twice the magnitude of the galactocentric acceleration. The more recent pulsar VLBI results of Deller et al. (2008) for PSR J0437-4715 likely strengthen this upper limit.

In the future it may be possible to search for the proper motion of the cores of quasars with uncertainties much smaller than $c$. Then it could be confirmed for the first time that the Hubble flow dominates the motion of quasar cores and that the velocity expected from the solar-system-barycenter acceleration is indeed to a high degree consistent with models.

\subsubsection{Nonlinear Motion of the Core within the Boundaries of the Proper-motion Limit}

Our observation of possibly significant jittery motion of the core within an area of the sky as small as $0.2 \times 0.2$ mas $^{2}$ would be only the second time such motion has been recorded unambiguously for a source with core-jet structure by using as a reference for the motion a physically unrelated source nearby on the sky. The first source for which such motion was detected is the core in the core-jet structure of the nearby galaxy M81. In this case, the center of the expanding shell of the nearby supernova 1993J was used as a reference (Bietenholz et al. 2001).

What caused this apparent nonlinear motion? Could such motion be indicative of orbital motion related to a binary black hole system? This possibility is unlikely since the motion is jittery and its magnitude too large to be physically reasonable.

Our measurements of the jittery motion of $\mathrm{C} 1$ along the north-south direction are within the noise determined from the jitter in the positions of B2250+194 relative to B2252+172. C1's jittery motion along the east-west direction is above the noise level. In fact, the peak-to-peak variation is 2.5 times larger than the corresponding variation in the positions of the two reference 
sources and may be significant. If so, it is likely caused by slight brightness-distribution changes due to activity at the foot of the jet close to the putative supermassive black hole. Such changes may have influenced the fit position of the Gaussian core component, C1 (Paper II).

This result, if confirmed, has implications for high-precision astrometric observations in general. It shows that any component, even a core component, clearly identified in the structure of a celestial object may still move on the sky.

\subsection{The Relevance for $G P-B$}

The goal of $G P-B$ at launch was to measure the precession of the gyroscopes relative to distant inertial space with a standard error of 0.5 mas $\mathrm{yr}^{-1}$ or less in each sky coordinate. To be a minor contributor to the error budget, the proper motion of the guide star, IM Peg, was to be determined with a standard error no larger than 0.14 mas $\mathrm{yr}^{-1}$ in each sky coordinate. Our reference source, the quasar 3C 454.3 was shown to be stationary within the CRF over $\sim 30$ years of geodetic VLBI observations to within 0.023 and 0.009 mas $\mathrm{yr}^{-1}$ in $\alpha$ and $\delta$, respectively. More to the point, our primary reference point for $G P-B, \mathrm{C} 1$ in $3 \mathrm{C} 454.3$, is stationary with respect to $\mathrm{B} 2250+194$ to within 0.038 and 0.035 mas $\mathrm{yr}^{-1}$ and within the CRF to within 0.046

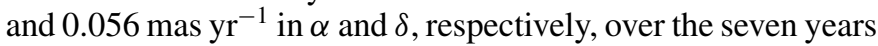
that almost entirely cover the period of our VLBI observations in support of the $G P-B$ mission and is therefore a negligible contributor to the error budget of the proper motion of the guide star.

\section{CONCLUSIONS}

Here we summarize our observations and data analysis, and give our conclusions.

1. We made differential VLBI observations at 35 epochs of the quasar 3C 454.3 and the radio galaxy B2250+194 along with 12 epochs of the extragalactic, unidentified source, B2252+172, at $8.4 \mathrm{GHz}$ between 1997 and 2005. With these sources we provided a reference frame composed of extragalactic sources nearby on the sky to IM Peg and, together with geodetic VLBI observations made by others we provided a (global) CRF, the latter closely linked to the ICRF2, for the determination of the proper motion of the $G P-B$ guide star IM Peg with respect to the distant universe.

2. We analyzed our differential VLBI observations using phase-referenced mapping and phase-delay fitting in combination with a Kalman filter.

3. Our $1 \sigma$ upper limit of the proper motion of $\mathrm{B} 2252+172$ relative to $\mathrm{B} 2250+194$ is $11 \mu \mathrm{as} \mathrm{yr}^{-1}$ in $\alpha$ and $24 \mu \mathrm{as} \mathrm{yr}^{-1}$ in $\delta$ for the time from 2002 to 2005 , identifying B2252+172 unequivocally as extragalactic and providing for a highly stable reference frame of sources nearby on the sky to $3 \mathrm{C} 454.3$.

4. Our $1 \sigma$ upper limits of the proper motions of 3C 454.3, $\mathrm{B} 2250+194$, and $\mathrm{B} 2252+172$ in the CRF determined with geodetic observations, and the latter two also partly with phase-delay observations, are $\leqslant 30 \mu$ as $\mathrm{yr}^{-1}$ in each coordinate.

5. Our $1 \sigma$ upper limit on the proper motion of $\mathrm{C} 1$, our core component of $3 \mathrm{C} 454.3$, relative to the combination of B2250+194 and B2252+172 for the time from 2002 to 2005 is $<35 \mu$ as $\mathrm{yr}^{-1}$ in each of $\alpha$ and $\delta$, indicating that $\mathrm{C} 1$ is highly stable with respect to two extragalactic sources nearby on the sky.
6. The $1 \sigma$ upper limit on the proper motion of $\mathrm{C} 1$ in the $\mathrm{CRF}$

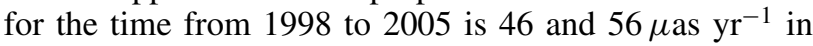
$\alpha$ and $\delta$, respectively. This is our fundamental result of the stationarity of the reference point for the guide star IM Peg in support of the $G P-B$ mission. For the shorter time from 2002 to 2005 the $1 \sigma$ upper limit on the proper motion of $\mathrm{C} 1$

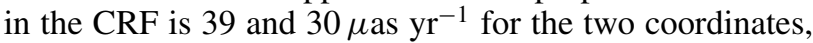
respectively, corresponding to subluminal motion of $\leqslant 1.0 \mathrm{c}$ and $<0.8 c$, for $\alpha$ and $\delta$, respectively, for an angular-size distance to $3 \mathrm{C} 454.3$ of $1.6 \mathrm{Gpc}$, for a flat universe with $H_{0}=70 \mathrm{~km} \mathrm{~s}^{-1} \mathrm{Mpc}^{-1}, \Omega_{M}=0.27$, and $\Omega_{\lambda}=0.73$.

7. The source coordinates of $\mathrm{C} 1$ in the CRF differ from those of 3C 454.3 determined from geodetic group-delay data by $131 \pm 98 \mu$ as in $\alpha$ and $-338 \pm 105 \mu$ as in $\delta$, the latter coordinate being different at the $3.2 \sigma$ level. This difference in $\delta$ is not understood, except possibly as a statistical fluke.

8. C2 and J1, the latter for the period from 2002 to 2005 , are stationary in the CRF to within bounds as small as those for C1. However, C1's flat spectrum and compactness in contrast to the spectra and the compactness of the other components indicate that $\mathrm{C} 1$ is closest to the putative supermassive black hole and the probable gravitational center of the quasar.

9. The jet components, D1, D2, and Jext clearly move in the CRF. Their motions correspond to superluminal speeds, which for D2 is $5 c$.

10. Notwithstanding our limit on the proper motion of $\mathrm{C} 1$, there is evidence for its having jittery $\sim 0.2$ mas east-west motion above the noise level, likely related to jet activity in the vicinity of the core. This evidence is consistent with the jittery motion of $\mathrm{C} 1$ found in Paper II.

11. The $1 \sigma$ upper limit on the parallax of $\mathrm{C} 1$ relative to $\mathrm{B} 2250+194$ and B2252+172 is $20 \mu \mathrm{as}$, one of the most, if not the most, accurate limit so far obtained, corresponding to an unsurprising lower limit of $50 \mathrm{kpc}$ on its distance from Earth and demonstrating the sensitivity of parallax measurements with VLBI.

12. The upper limit on the proper motion of $3 \mathrm{C} 454.3$ over $\sim 30$ years of geodetic VLBI observations and of $\mathrm{C} 1$ over $\sim 8.5$ years of our phase-delay VLBI observations is sufficiently small to meet the goal of the $G P-B$ mission and therefore to justify use of $\mathrm{C} 1$ as the primary reference point for $G P-B$.

This research was primarily supported by NASA, through a contract with Stanford University to SAO, and a subcontract from SAO to York University. The National Radio Astronomy Observatory is a facility of the National Science Foundation operated under cooperative agreement by Associated Universities, Inc. The DSN is operated by JPL/Caltech, under contract with NASA. This research has made use of the United States Naval Observatory (USNO) Radio Reference Frame Image Database (RRFID). We have made use of NASA's Astrophysics Data System Abstract Service, developed and maintained at SAO. Jeff Cadieux and Julie Tome helped with the data reduction during their tenure as students at York University. We thank the International VLBI Service for Geodesy and Astrometry (IVS; Schlüter \& Behrend 2007) for their support.

\section{REFERENCES}

Bartel, N., Herring, T. A., Ratner, M. I., Shapiro, I. I., \& Corey, B. E. 1986, Nature, 319, 733

Bietenholz, M. F., Bartel, N., Lebach, D. E., et al. 2012, ApJS, 201, 7 (Paper VII) 
Bietenholz, M. F., Bartel, N., \& Rupen, M. P. 2001, ApJ, 557, 770

Bietenholz, M. F., Bartel, N., \& Rupen, M. P. 2004, ApJ, 615, 173

Brunthaler, A., Reid, M. J., Falcke, H., Greenhill, L. J., \& Henkel, C. 2005, Science, 307, 1440

Campbell, R. M. 1999, New Astron. Rev., 43, 617

Cawthorne, T. V., \& Gabuzda, D. C. 1996, MNRAS, 278, 861

Charlot, P., Boboltz, D. A., Fey, A. L., et al. 2010, AJ, 139, 1713

Condon, J., Darling, J., Kovalev, Y. Y., \& Petrov, L. 2011, arXiv:1110.6252

Deller, A. T., Verbiest, J. P. W., Tingay, S. J., \& Bailes, M. 2008, ApJ, 685, L67

Fey, A. L., Gordon, D., \& Jacobs, C. S. (ed.) 2009, IERS Technical Note No. 35 (Frankfurt: Verlag des Bundesamts für Kartographie und Geodäsie), http://www.iers.org/IERS/EN/Publications/TechnicalNotes/tn35.html

Fomalont, E. B., \& Kopeikin, S. M. 2003, ApJ, 598, 704

Gómez, J.-L., Marscher, A. P., \& Alberdi, A. 1999, ApJ, 522, 74

Jorstad, S. G., Marscher, A. P., Lister, M. L., et al. 2005, AJ, 130, 1418

Jorstad, S. G., Marscher, A. P., Mattox, J. R., et al. 2001, ApJS, 134, 181

Kantowski, R., Kao, J. K., \& Thomas, R. C. 2000, ApJ, 545, 549

Kovalev, Y. Y., Lobanov, A. P., Pushkarev, A. B., \& Zensus, J. A. 2008, A\&A, 483, 759

Lanyi, G. E., Boboltz, D. A., Charlot, P., et al. 2010, AJ, 139, 1695

Lebach, D. E., Bartel, N., Bietenholz, M. F., et al. 2012, ApJS, 201, 4 (Paper IV)

Lebach, D. E., Ratner, M. I., Shapiro, I. I., et al. 1999, ApJ, 517, L43

Lestrade, J.-F., Preston, R. A., Jones, D. L., et al. 1999, A\&A, 344, 1014

Marcaide, J. M., \& Shapiro, I. I. 1983, AJ, 88, 1133

Martí-Vidal, I., Marcaide, J. M., Guirado, J. C., Pérez-Torres, M. A., \& Ros, E. 2008, A\&A, 478, 267
Pagels, A., Krichbaum, T. P., Graham, D. A., et al. 2004, in Proc. of European VLBI Network 7th Symp. on New Developments in VLBI Science and Technology, ed. R. Bachiller et al. (Madrid: Observatorio Astronòmico Nacional), 7

Pauliny-Toth, I. I. K. 1998, in ASP Conf. Ser. 144, IAU Colloq. 164, Radio Emission from Galactic and Extragalactic Compact Sources, ed. J. A. Zensus, G. B. Taylor, \& J. M. Wrobel (San Francisco, CA: ASP), 75

Pauliny-Toth, I. I. K., Porcas, R. W., Zensus, J. A., Kellermann, K. I., \& Wu, S. Y. 1987, Nature, 328, 778

Petrov, L., Gordon, D., Gipson, J., et al. 2009, J. Geod., 83, 859

Porcas, R. W. 2009, A\&A, 505, L1

Pradel, N., Charlot, P., \& Lestrade, J.-F. 2006, A\&A, 452, 1099

Ransom, R. R., Bartel, N., Bietenholz, M. F., et al. 2012a, ApJS, 201, 2 (Paper II)

Ransom, R. R., Bartel, N., Bietenholz, M. F., et al. 2012b, ApJS, 201, 6 (Paper VI)

Ratner, M. I., Bartel, N., Bietenholz, M. F., et al. 2012, ApJS, 201, 5 (Paper V)

Rioja, M. J., \& Porcas, R. W. 2000, A\&A, 355, 552

Schlüter, W., \& Behrend, D. 2007, J. Geod., 81, 379

Shapiro, I. I., Bartel, N., Bietenholz, M. F., et al. 2012, ApJS, 201, 1 (Paper I)

Shapiro, I. I., Wittels, J. J., Counselman, C. C., III., et al. 1979, AJ, 84, 1459

Sokolovsky, K. V., Kovalev, Y. Y., Pushkarev, A. B., \& Lobanov, A. P. 2011, A\&A, 532, A38

Sovers, O. J., Fanselow, J. L., \& Jacobs, C. S. 1998, Rev. Mod. Phys., 70, 1393

Titov, O. 2010, MNRAS, 407, L46

Titov, O., Lambert, S. B., \& Gontier, A.-M. 2011, A\&A, 529, A91

Vercellone, S., D’Ammando, F., Vittorini, V., et al. 2010, ApJ, 712, 405

Villata, M., Raiteri, C. M., Balonek, T. J., et al. 2006, A\&A, 453, 817

Zakamska, N. L., \& Tremaine, S. 2005, AJ, 130, 1939 